\title{
Anesthesia Management of Liver Transplantation
}

\author{
Yoogoo Kang and Elia Elia
}

\section{Contents}

Introduction

Anatomy of the Liver

Hepatic Function

Carbohydrate Metabolism

Lipid Metabolism ..................

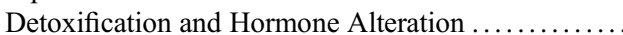

Excretory Function ..............................

Filtration Function

Pathophysiology of Liver Cirrhosis

Central Nervous System

Cardiovascular System

Pulmonary System

Renal System ....

Coagulation System

Anesthesia Consultation

Cardiovascular Assessment

Pulmonary Evaluation

Renal Function ....

Fulminant Hepatic Failure

Coagulation System

Contraindications

Candidate Selection

Surgical Aspects of Liver Transplantation

Orthotopic Liver Transplantation (OLT) with Simple

Venous Cross-Clamping

OLT with Venovenous Bypass ....

Y. Kang $(\bowtie) \cdot$ E. Elia

Department of Anesthesiology, Sidney Kimmel Medical

College, Thomas Jefferson University Hospital,

Philadelphia, PA, USA

e-mail: Yoogoo.kang@jefferson.edu; Elia.Elia@jefferson. edu

(C) Springer International Publishing Switzerland 2016

C. Doria (ed.), Contemporary Liver Transplantation,

DOI 10.1007/978-3-319-05543-5 9-1

Piggyback Technique $\ldots \ldots \ldots \ldots \ldots \ldots \ldots \ldots \ldots \ldots, 18$

2
Preparation and Anesthetics .................... 19

\section{Physiologic Homeostasis During Liver}

Transplantation ......................... 21

Cardiovascular Homeostasis ................... 22

Pulmonary Homeostasis ....................... 25

Cerebral Homeostasis ........................... 26

Coagulation .................................. 26

Electrolyte and Acid-Base Homeostasis ............ 28

Metabolic Homeostasis ....................... 30

Renal Homeostasis ............................. 31

Conclusion of Surgery ..................... 31

Postoperative Complications ................... 31

Hepatic Complications ....................... 31

Extrahepatic Complications .................. 32

Anesthesia for Specific Conditions ............. 34

Retransplantation of the Liver .................. 34

Pediatric Liver Transplantation ................. 35

Live-Donor Hepatectomy ...................... 35

Surgery After Liver Transplantation .............. 36

Cadaveric Donor Procurement .................. 36

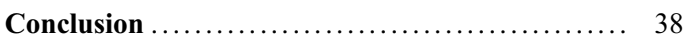

Cross-References ........................... 38

References .............................. 39

\section{9}

1

(1)

5

6

(2)




\section{Abstract}

Anesthesia for liver transplantation pertains to a continuum of critical care of patients with end-stage liver disease. Hence, anesthesiologists, armed with a comprehensive understanding of pathophysiology and physiologic effects of liver transplantation on recipients, are expected to maintain homeostasis of all organ function. Specifically, patients with fulminant hepatic failure develop significant changes in cerebral function, and cerebral perfusion is maintained by monitoring cerebral blood flow and cerebral metabolic rate of oxygen, and intracranial pressure. Hyperdynamic circulation is challenged by the postreperfusion syndrome, which may lead to cardiovascular collapse. The goal of circulatory support is to maintain tissue perfusion via optimal preload, contractility, and heart rate using the guidance of right-heart catheterization and transesophageal echocardiography. Portopulmonary hypertension and hepatopulmonary syndrome have high morbidity and mortality, and they should be properly evaluated preoperatively. Major bleeding is a common occurrence, and euvolemia is maintained using a rapid infusion device. Pre-existing coagulopathy is compounded by dilution, fibrinolysis, heparin effect, and excessive activation. It is treated using selective component or pharmacologic therapy based on the viscoelastic properties of whole blood. Hypocalcemia and hyperkalemia from massive transfusion, lack of hepatic function, and the postreperfusion syndrome should be aggressively treated. Close communication between all parties involved in liver transplantation is also equally valuable in achieving a successful outcome.

\section{Keywords}

Anesthesia - Cirrhosis - Coagulation - Liver transplantation - Fibrinolysis • Hepatopulmonary syndrome - Hypocalcemia

- Hyperkalemia - Physiology • Portopulmonary hypertension

Postreperfusion syndrome - Rapid infusion device - Transesophageal echocardiography • Thromboelastography

\section{Introduction}

Dr. Thomas Starzl of Denver, Colorado, USA, who believed that "liver transplantation is an effective treatment providing exactly what is needed for patients with end-stage liver disease (ESLD)," performed the first successful orthotopic liver transplantation (OLT) in a 3-year-old boy with biliary atresia in 1963 (Starzl et al. 1963). During the first two decades of the procedure's history, liver transplantation led by Starzl and Sir Roy Calne of Cambridge encountered almost insurmountable challenges, including complexity of surgical technique, primitive anesthesia and intensive care, less-than-adequate immunosuppression and organ preservation, and devastating infection. The number of procedures performed was relatively few, and the success rate was low. However, their keen observations on these early clinical experiences laid the foundation of modern liver transplantation (Starzl and Putnam 1969; Calne 1983).

Breakthroughs were made in each decade following the first transplantation. In the 1980s, venovenous bypass was introduced to maintain better hemodynamic stability (Shaw et al. 1984), cyclosporine was found to be a superior immunosuppressant to azathioprine, and anesthesiologists answered important clinical questions, including those relating to the monitoring and treatment of coagulopathy, hemodynamic changes, and the role of the electrolyte imbalance. In the 1990s, FK506 (tacrolimus) became the immunosuppressant of choice (Starzl et al. 1989), University of Wisconsin solution was introduced to extend the safe cold ischemia time to $24 \mathrm{~h}$ (Kalayoglu et al. 1988), and the piggyback technique simplified surgery in select patients (Tzakis et al. 1989). In the past 15 years, liver transplantation has been performed in most major medical centers with a 1-year survival rate of greater than $85 \%$, and living donor liver transplantation has become a valuable alternative. 
Fig. 1 Segments of the liver (Reprinted from Clin Liver Dis, 4, Ghobrial RM, Amersi F, Busuttil RW, Surgical advances in liver transplantation. Living related and split donors, 553-565, Copyright (2000), with permission from Elsevier)

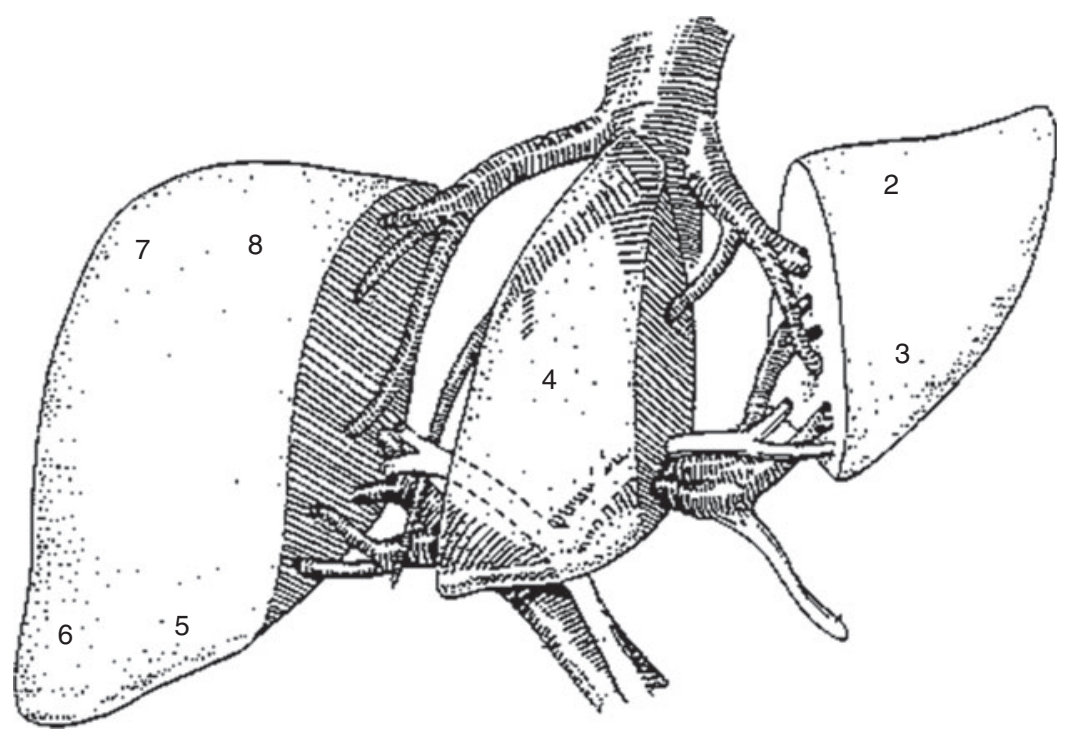

Liver transplantation requires a true multidisciplinary approach, and anesthesiologists and intensivists have played a major role in the successful outcome of liver transplantation. In support of the important role of anesthesiologists in liver transplantation, the American Society of Anesthesiologists (ASA) developed the Guidelines for Director of Liver Transplant Anesthesia in 2001. The guidelines specified that the Director should have fellowship training in critical care medicine, cardiac anesthesiology, or transplantation anesthesiology that includes the perioperative care of at least ten liver transplant recipients or experience in the perioperative care of at least 20 liver transplant recipients in the operating room. In addition, the Director is expected to obtain a minimum of $8 \mathrm{~h}$ of Accreditation Council for Continuing Medical Education (ACCME) Category I continuing medical education (CME) credit in transplantationrelated educational activities within the most recent 3-year period.

In this chapter, physiology and pathophysiology of liver disease and anesthesia care of liver transplantation are described based on clinical experience at the University of Pittsburgh (Pittsburgh, PA, USA) and Thomas Jefferson University (Philadelphia, PA, USA).

\section{Anatomy of the Liver}

The liver, which weighs $1200-1500 \mathrm{~g}$ in adults, is traditionally divided into the right and left lobe in reference to the location of the falciform ligament. Couinaud, however, divided the liver into the right and left hemiliver using the Cantlie's line, which extends from the inferior vena cava (IVC) to the gall bladder, and each hemiliver is further divided into four segments (Couinaud 1954). The left hemiliver is composed of the traditional left lobe along with the caudate and quadrate lobe. Liver resections based on these segmental definitions are right hepatectomy (segments 5-8), right lobectomy (segments 4-8), left hepatectomy (segments 1-4), and left lobectomy (segments 1-3) (Fig. 1) (Bismuth 1982).

The liver has a unique dual blood supply: arterial supply from the hepatic artery, a branch of the celiac axis, and venous supply from the portal vein formed by the union of the splenic and superior mesenteric vein. Despite liver mass constituting only $2.5 \%$ of the total body weight, the total hepatic blood flow is approximately $100 \mathrm{~mL} /$ $100 \mathrm{~g} / \mathrm{min}$, or $25 \%$ of cardiac output. The hepatic artery supplies approximately $25-30 \%$ of hepatic blood flow and $45-50 \%$ of the oxygen 
requirement, while the portal vein supplies $70-75 \%$ of hepatic blood flow and $50-55 \%$ of oxygen. The venous drainage is through the right, middle, and left hepatic veins, which merge into the IVC. The valveless portal vein is a low pressure/low resistance circuit, while the hepatic artery is a high pressure/high resistance system. Hepatic blood flow is primarily regulated by local metabolic demand with an inverse relationship between portal venous and hepatic arterial flow: an increase in the hepatic adenosine level triggered by a reduced portal venous flow increases hepatic arterial blood flow (Gelman and Ernst 1977; Lautt et al. 1985). The hepatic artery buffer response appears to be functional even after liver transplantation (Payen et al. 1990), and this response may be responsible for the development of the small-for-size syndrome after living donor or split liver transplantation (Kiuchi et al. 1999). Small-for-size syndrome develops in a patient who received a donor graft that was less than $1 \%$ of the recipient's body weight and is caused by decreased hepatic arterial flow in response to increased portal venous flow and pressure. Subsequently, a prolonged postoperative reduction in hepatic arterial flow can lead to centrilobular tissue necrosis, biliary ischemia, and hepatic arterial thrombosis (Smyrniotis et al. 2002). There is no buffer response in the portal system because the portal vein cannot regulate its blood flow. Therefore, alterations in the hepatic arterial blood flow do not induce compensatory changes in the portal blood flow (Lautt 1983).

The mean pressure in the hepatic artery is similar to that in the aorta, while portal vein pressure ranges between 6 and $10 \mathrm{mmHg}$. The portal pressure depends primarily on the degree of constriction or dilatation of the splanchnic arterioles and on intrahepatic resistance. Both afferent systems merge at the sinusoidal bed, where the pressure is estimated to be $2-4 \mathrm{mmHg}$ higher than that in the IVC. The liver serves as a blood reservoir, and it replenishes blood volume of up to $25 \%$ rapidly in the case of an acute bleeding episode (Lautt 2007). Hepatic blood volume may expand considerably in cardiac failure by venous congestion.

The liver is innervated by the left and right vagi, the right phrenic nerve, and fibers from the T7-T10 sympathetic ganglia. The hepatic artery is innervated mainly by sympathetic fibers, and hepatocytes, by the unmyelinated sympathetic fibers. The bile ducts are innervated by both sympathetic and parasympathetic fibers. The role of hepatic innervation is unclear, as denervation of the transplanted liver does not affect its function (Kjaer et al. 1994).

Bile flow begins from the bile canaliculi to the common bile duct. Hepatic lymph forms in the space between the sinusoid and the hepatocyte (space of Disse) and flows to lymph nodes in the hilum and IVC. The transdiaphragmatic lymphatic flow is the cause of pleural effusions in the presence of large ascites.

The liver is made of parenchymal cells (hepatocytes) and non-parenchymal cells (sinusoidal endothelial, Kupffer, stellate, dendritic, and lymphocyte). Hepatocytes make up $60-80 \%$ of liver cells and carry out hepatic metabolic, synthetic, and detoxification functions. Polyhedral hepatocytes are arranged in one-cell thick plates with endothelium-lined sinusoids on both sides. Each hepatocyte cell membrane has three distinct membrane domains. The sinusoidal membrane is adjacent to the sinusoidal endothelium and has numerous microvilli abutting into the space of Disse. Fenestrae within the sinusoidal endothelium without the basement membrane permit intimate contact between sinusoidal blood and the hepatocytes to allow the passage of big molecules, including lipoproteins. Liver sinusoidal endothelial cells make up 15-20\% of liver cells and release nitric oxide to regulate vascular resistance. They are, along with dendritic cells and lymphocytes, part of the innate immune system. The space of Disse contains phagocytic Kupffer cells that participate in the hepatic inflammatory process. The Ito cells, also known as stellate cells, are the major site of vitamin A storage, and their activation results in hepatic fibrosis and cirrhosis. Reticulin fibers in the space of Disse support the 
Fig. 2 Schematic diagram of the acinus

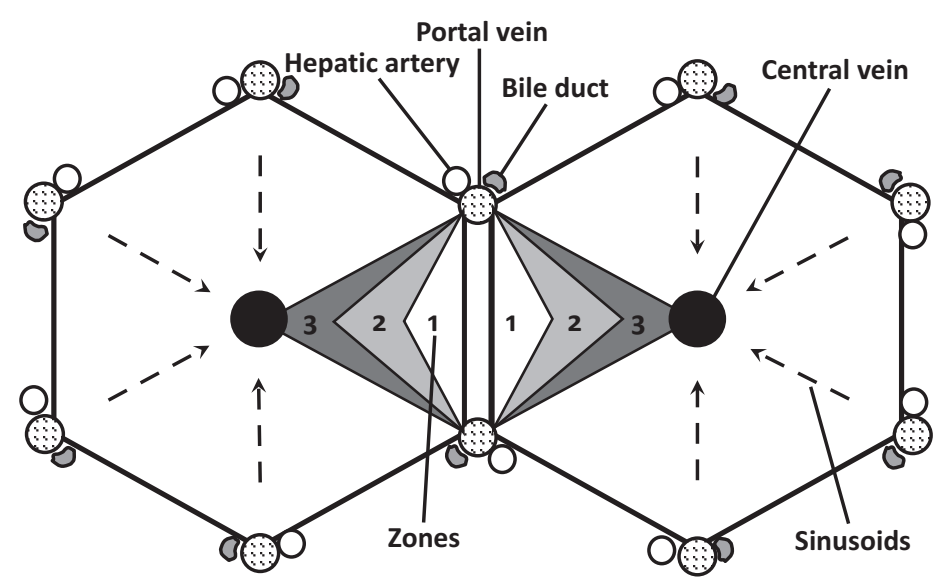

sinusoidal framework, and weakening of these supporting fibers results in rupture of sinusoidal walls and formation of blood-filled cysts known as peliosis hepatis, a forerunner of cirrhosis. The apical membrane circumscribes the canaliculus, the earliest component of the biliary system. The lateral hepatic membrane is found between adjacent hepatocytes.

The functional unit of the liver is the acinus. Terminal portal veins communicate with terminal hepatic venules, with sinusoids bridging the gap between the two vessels (Fig. 2). Each sinus contains three zones with equal blood pressure and oxygen content. The periportal zone (Zone 1) receives blood highest in oxygen content and the pericentral or perivenular zone (Zone 3 ) receives blood lowest in oxygen content. As a result, the hepatocytes in the perivenular zone (Zone 3) are more vulnerable to ischemic damage and nutrient depletion. Oxidative and reductive functions are predominantly performed by hepatocytes at the periportal zone and glucuronidation is performed by those at the perivenular zone, although hepatocytes of the two different zones are functionally integrated (Lamers et al. 1989). The unique structure of the liver acinus is well-suited for bidirectional transfer of nutrients. The low pressure in the portal venous system allows blood to flow slowly through the sinusoids. Hepatic arterial blood flows mainly to the terminal bile canaliculi, although it augments sinusoidal flow to give a gentle pulsatility. In patients with liver cirrhosis, the sinusoids acquire features of systemic capillaries: the space of Disse widens with collagen deposits at the basement membrane, endothelial fenestrations become smaller and fewer, and hepatic microvilli efface. All of these changes reduce transport across the sinusoidal walls and result in hepatic dysfunction. Furthermore, widespread fibrosis and scarring reduce the number and size of the small portal and hepatic veins and increase intrahepatic vascular resistance to the development of portal hypertension (Popper 1977). The sluggish blood flow in the altered vascular architecture promotes thrombosis, causing further cell necrosis and fibrosis (Wanless et al. 1995).

The liver undergoes rapid regeneration through proliferation of hepatocytes to maintain the critical mass necessary for normal liver function. For example, the newly transplanted hemiliver from a living related donor regenerates to about $85 \%$ of its original whole liver size in 7-14 days. The major hepatic growth factors are epidermal growth factor and hepatocyte growth factor (Michalopoulos 1990). Administration of the epidermal or hepatocyte growth factor to normal rats, however, does not cause hepatocyte replication. This negative response suggests that liver regeneration involves a two-step process: the initial signal generated by an acute increase in metabolic demand associated with the loss of hepatocytes triggers a set of early response genes that prime hepatocytes to respond to various growth factors. 
In apoptosis or programmed cell death, aging hepatocytes are removed and new cells are produced in a continuous manner (Ellis et al. 1991).

\section{Hepatic Function}

The liver has three major functions: metabolism, bile production and secretion, and filtration of harmful substances.

\section{Carbohydrate Metabolism}

The principal role of the liver is to provide the body with normal glucose levels, which are regulated by insulin, glucagon, growth hormone, and catecholamines (Pilkis and Granner 1992). The liver converts glucose into glycogen (glycogenesis) and utilizes glucose for the synthesis of fatty acids.

Cirrhotic patients are frequently hyperglycemic although their insulin level is elevated (Petrides and DeFronzo 1989). This insulin resistance is caused by multiple mechanisms. Cirrhotic patients have an increased basal metabolic rate and use preferentially fatty acids as an energy source. Reduced glucose uptake and limited glucose storage in the liver and muscle lead to hyperglycemia. Other contributing factors are increased serum fatty acids, which inhibit glucose uptake by muscle; altered second messenger activity after insulin binding to its receptors; an increased concentration of serum cytokines associated with elevated levels of endotoxins; and increased levels of glucagon and catecholamines.

\section{Protein and Amino Acid Metabolism}

The liver is the major organ for protein synthesis, and albumin is the most important protein product. Albumin is the major contributor to plasma oncotic pressure and binds and transports bilirubin, hormones, fatty acids, and other substances. Hypoalbuminemia is commonly caused by decreased hepatic synthetic function, although it can be secondary to an enlarged volume of distribution, reduced level of amino acid precursors, and losses into the urine, peritoneum and pleural cavity, and leads to peripheral edema, ascites, and pleural effusions. The low serum oncotic pressure stimulates the hepatic albumin synthesis in healthy subjects, but this is impaired in patients with cirrhosis (Pierrangelo et al. 1992). The liver synthesizes all coagulation factors (except von Willebrand factor) and protein $\mathrm{C}$ and S. Factors II, VII, IX, and X undergo a posttranslational vitamin $\mathrm{K}$-dependent modification involving $\gamma$-carboxylation of specific glutamic acid residues in the liver.

The liver is the primary site of interconversion of amino acids. Anabolic processes synthesize proteins from amino acids, while catabolic processes convert amino acids either to keto acids by transamination or ammonia by oxidative deamination. Ammonia, in turn, is converted to urea by the Krebs-Henseleit cycle. In patients with liver disease, derangement of both anabolic and catabolic processes results in decreased production of blood urea nitrogen (BUN) and accumulation of ammonia, a contributing factor in the development of hepatic encephalopathy. The liver produces acute-phase reactants, such as $\alpha$-fetoprotein, ceruloplasmin, fibrinogen, transferrin, complement, and ferritin. They are expressed during acute and chronic systemic inflammation, and their activation is mediated by interleukin-6, tumor necrosis factor, interferon- $\gamma$, and glucocorticoids.

\section{Lipid Metabolism}

The liver takes up fatty acids and cholesterol from diet and peripheral tissues to produce and release lipoprotein complexes into circulation. Fatty acids released from adipocytes are bound to serum albumin and transported to the liver for the synthesis of phospholipids and triglycerides. The liver produces fatty acids from small molecular weight precursors, and cholesterol synthesis is regulated by the rate-limiting enzyme 3-hydroxyl-3 methylglutaryl coenzyme A reductase (HMG-CoA reductase). Lipids are exported out of the liver by very low-density lipoprotein 
(VLDL) particles, which are the major carriers of plasma triglycerides during non-absorptive states. Lipids are temporarily stored in the liver as fat droplets, or as cholesteryl esters in the case of cholesterol, and are directly excreted into bile or metabolized into bile acids. The liver is the major site for sterol excretion and production of bile acids.

Various abnormalities in lipid metabolism are common in liver disease. Hypertriglyceridemia $(250-500 \mathrm{mg} / \mathrm{dL})$ is the most common presentation and may be caused by decreased synthesis of lipoproteins, decreased hepatic clearance of lipoprotein complexes, or re-entry of biliary content into the serum. Alcoholic liver injury results in increased fatty acid synthesis and steatosis (Lieber 1993). Paradoxically, an increased high-density lipoprotein (HDL) 3 level has been noted with moderate alcohol consumption, which may explain the reduced risk of atherosclerosis in these patients (Chait and Brunzell 1990). Patients with cholestatic liver diseases have elevated total serum cholesterol and triglycerides because the bile is rich in cholesterol, phospholipids, and lecithin.

\section{Detoxification and Hormone Alteration}

The liver eliminates drugs through two types of reactions. The phase 1 reactions include oxidation, reduction, hydroxylation, sulfoxidation, deamination, dealkylation, and methylation of reactive substances. These reactions involve systems such as cytochrome P450 and typically occur in the periportal area of the liver. The phase 2 reactions, which transform lipophilic agents into more water-soluble compounds, take place in the pericentral area. In patients with liver disease, hepatic drug clearance is usually reduced due to the enlarged volume of distribution and decreased hepatic metabolism. As a result, a large initial dose of medications followed by small, titrated maintenance doses are required to achieve the desired pharmacologic effects.

Several hormones are deactivated or altered in the liver. The deactivated hormones are insulin, glucagon, steroid hormones, aldosterone, thyroxine, and triiodothyronine. The liver converts testosterone into androsterone and estrogen into estrone and estriol. Abnormal levels of estrogen and testosterone in patients with liver disease lead to testicular atrophy, loss of pubic and axillary hair, spider angioma, and gynecomastia.

\section{Excretory Function}

The liver removes various substances from the body, and bile formation is one of the most important excretory functions. When membranes of old erythrocytes rupture, the released hemoglobin is taken up by the reticuloendothelial cells and is split into heme and globin. Heme converts to biliverdin, which, in turn, is reduced to free bilirubin and released into the plasma. The free bilirubin-albumin complex is taken up by the hepatocytes. Bilirubin conjugates primarily with glucuronic acid and is actively transported into the bile. A small portion of conjugated bilirubin returns to the plasma directly from the sinusoids or indirectly by absorption from the bile ducts and lymphatics. Bilirubin is converted into urobilinogen by the intestinal bacterial flora. Some urobilinogen is reabsorbed through the intestinal mucosa and is re-excreted into the intestine. Bile acids, which enhance absorption of vita$\min \mathrm{K}$, are also excreted into the bile by the liver.

\section{Filtration Function}

The liver, located between the splanchnic and systemic venous system, acts as a vascular filter. Kupffer cells phagocytose immune complexes, endotoxins, and bacteria in the portal venous blood and process antigens for presentation to immunocompetent cells. The liver also removes activated coagulation elements from circulation to prevent excessive coagulation or fibrinolysis.

\section{Pathophysiology of Liver Cirrhosis}

Liver cirrhosis is defined as progressive fibrosis and the formation of regenerative nodules, and is the final common pathway in which hepatocytes 
Table 1 Child-Pugh score

\begin{tabular}{|c|c|c|c|}
\hline \multirow[b]{2}{*}{ Presentation } & \multicolumn{3}{|l|}{ Points } \\
\hline & 1 & 2 & 3 \\
\hline Albumin (g/dL) & $>3.5$ & $2.8-3.5$ & $<2.8$ \\
\hline \multicolumn{4}{|l|}{ Prothrombin time } \\
\hline Seconds prolonged & $<4$ & $4-6$ & $>6$ \\
\hline INR & $<1.7$ & $1.7-2.3$ & $>2.3$ \\
\hline \multicolumn{4}{|l|}{ Bilirubin (mg/dL) } \\
\hline Hepatocellular disease & $<2$ & $2-3$ & $>3$ \\
\hline Cholestatic disease & $<4$ & $4-10$ & $>10$ \\
\hline Ascites & Absent & Mild-moderate & Tense \\
\hline Encephalopathy & None & Grade $1-2$ & Grade $3-4$ \\
\hline
\end{tabular}

Class $\mathrm{A}=5-6$ points, $\mathrm{B}=7-9$ points, and $\mathrm{C}=10-15$ points

INR international normalized ratio

are replaced by connective tissue after various, repetitive insults. The amount of remaining functional hepatic mass and the degree of architectural distortion determine the functional state of the liver. Portal hypertension is inevitable in advanced cirrhosis and leads to ascites, variceal bleeding, and encephalopathy. The severity of cirrhosis is frequently classified using the ChildPugh score (Table 1), and a score of $>6$ suggests a short life expectancy.

\section{Central Nervous System}

Hepatic encephalopathy is a reversible neuropsychiatric condition in both acute and chronic liver failure. In chronic liver disease, hepatic encephalopathy develops in $28 \%$ of patients within 10 years of compensated cirrhosis and is associated with spontaneously developed or surgically created portosystemic shunting (Butterworth 2001). The degree of encephalopathy is stratified by a coma scale: Grade 1, subtle confusion; Grade 2, somnolence; Grade 3, unconsciousness with response to pain stimulation; and Grade 4, deep coma. Clinically, asterixis, flapping tremor, and fetor hepaticus (musty, sweet breath odor) are confirmatory of hepatic encephalopathy.

The main cause of hepatic encephalopathy is the altered expression of several genes for various neurotransmitter proteins in the brain (Butterworth 2001). Decreased expression of the glutamate transporter (GLT-1) increases extracellular brain glutamate. An increased expression occurs in some receptors: monoamine oxidase increases degradation of monoamine transmitters, the peripheral-type benzodiazepine receptor increases inhibitory neurosteroids, and neuronal nitric oxide synthase increases nitric oxide production. Although its plasma level is not closely related to the severity of encephalopathy, ammonia is still considered to be a major contributing factor. Ammonia and manganese are known to alter the expression of the peripheraltype benzodiazepine receptor and neuronal nitric oxide synthase in exposed cells (Warskulat et al. 2001).

Magnetic resonance spectroscopy reveals brain edema and increased brain glutamine/glutamate in the frontal and parietal lobes; histologic findings are swelling and glycogen deposition in astrocytes. These changes in the brain coincide with impairment in the visuopractic capacity, visual scanning, and perceptual-motor speed on neuropsychiatric testing (Tarter et al. 1984). Subclinical hepatic encephalopathy can be detected by having patients perform a simple timed connect-the-numbers test.

Treatment of hepatic encephalopathy is based on the ammonia-lowering strategy, such as protein restriction, oral non-absorbable antibiotics, and lactulose. Rifaximin reduces the plasma ammonia level by destroying intestinal bacteria that produce urease. Metronidazole ( $800 \mathrm{mg} / \mathrm{day})$ is another antibiotic, although its adverse effects limit its use to 1 week at a time. Lactulose is a substrate 
for gut bacteria and reduces the formation of ammonia by lowering intestinal $\mathrm{pH}$. Rifaximin and lactulose are commonly used together, and the antibiotic is discontinued once eradication of disaccharide-metabolizing intestinal bacteria is indicated by an increase in stool $\mathrm{pH}$. Oral or parenteral ornithine aspartate, a substrate for the conversion of ammonia to urea and glutamine, has effects similar to those of lactulose, but with fewer adverse effects. In patients with severe encephalopathy, the molecular-absorbent recycling system (MARS) may be utilized to remove small and middle molecular weight water-soluble substances (Sorkine et al. 2001). The system appears to increase blood pressure and systemic vascular resistance, possibly by removing nitric oxide.

In fulminant hepatic failure, progressive hepatic coma is accompanied by a gradual increase in cerebral blood flow and intracranial pressure (ICP) (see Chapter $15 \triangleright$ Fulminant Hepatic Failure: Diagnosis and Management). Subsequently, vasogenic cerebral edema and severe intracranial hypertension develop and approximately $30-50 \%$ of patients die of brain herniation. Monitoring of ICP using a Ladd epidural sensor is useful in detecting intracranial hypertension, monitoring the therapeutic effects, and identifying patients who would survive after transplantation without neurologic damage (Lidorsky et al. 1992). Non-invasive neurologic assessment includes transcranial Doppler (TCD) to measure cerebral bloodflow velocity, determination of the cerebral metabolic rate for oxygen by calculating the oxygen content difference between arterial and jugular bulb venous blood, evoked potentials, and serial computed tomography (CT) scans (Aggarwal et al. 1994). Treatment includes osmotic and loop diuretics, barbiturateinduced coma, and hypothermia. The definitive treatment is usually transplantation.

\section{Cardiovascular System}

The presence of hyperdynamic circulation with a markedly increased cardiac output and decreased systemic vascular resistance was first described by Kowalski and Abelmann in the early 1950s
(Kowalski and Abelmann 1953). Several hypotheses have been proposed to explain this phenomenon, including an overactive sympathetic nervous system, inadequate clearance of vasoactive substances by the diseased liver, the presence of arteriovenous shunts, nitric oxide-induced vasodilation, and relative hypoxia in peripheral tissues (Benoit et al. 1984; Yokoyama et al. 1989; Kalb et al. 1993; D’Souza et al. 1993).

Although cardiac output is frequently two to three times normal, impaired systolic and diastolic function together with attenuated cardiac responsiveness to stimuli suggests that cardiomyopathy is present in cirrhotics (cirrhotic cardiomyopathy) (Lee 1989). Caramelo et al. noted a $50 \%$ decrease in cardiac output with volume expansion in a $\mathrm{CCl}_{4}$-induced cirrhotic rat model (Caramelo et al. 1986). In another rat model, the chronotropic response to isoproterenol was attenuated compared with that in control animals (Lee et al. 1990). Cardiac response to physical exercise is blunted in patients with cirrhosis, indicated by alterations in the pre-ejection period, isometric contraction time, and ratio of the pre-ejection period to left ventricular ejection time. In addition, abnormalities in myocardial diastolic indices suggest non-compliant ventricles. Histologically, myocardial fibrosis, mild subendocardial edema, and vacuolation of myocyte nucleus and cytoplasms are observed. The development of cirrhotic cardiomyopathy is multifactorial. It appears that the $\beta$-receptor system, the main stimulant of the ventricle, is dysfunctional. In humans, lymphocyte $\beta$-receptor density, which reflects cardiac $\beta$-receptor status, is reduced in patients with severe ascites (Gerbes et al. 1986), and $\beta$-receptor density of the cardiomyocyte sarcolemmal plasma membrane is reduced in cirrhotic rats (Liu and Lee 1999). Further, the $\beta$-receptor signal transduction pathway is impaired at several levels (Ma et al. 1996). Although cardiac contractile impairment may result from overactivity of the muscarinic $\mathrm{M}_{2}$ receptor, the receptor density and binding affinity are unchanged, suggesting normal parasympathetic function (Jaue et al. 1997). High serum catecholamine levels, a result of desensitization and down-regulation of $\beta$-receptors, may lead to myocardial dysfunction 
in the presence of $\alpha$-mediated coronary vasoconstriction. Additionally, overproduction of nitric oxide inhibits $\beta$-receptor-stimulated cyclic adenosine monophosphate (cAMP) release, causing myocardial dysfunction and vasodilation (Hare and Colucci 1995).

Coronary artery disease (CAD) was previously believed to be relatively uncommon in patients with cirrhosis as a result of generalized vasodilation and elevated levels of HDL and estrogen. In addition, autopsy findings showed relatively fewer atherosclerotic changes and myocardial infarction. However, studies have shown that CAD is not uncommon, and moderate-to-severe CAD was found in approximately $27 \%$ of patients who underwent coronary artery catheterization as a part of liver transplantation workup (Carey et al. 1995). In another study of 161 liver transplantation candidates who were at risk for CAD and referred for coronary angiography, $25 \%$ of patients had at least one moderate or severe $(>50 \%)$ coronary stenosis (TiukinhoyLaing et al. 2006).

Endocarditis is three times more common in patients with liver disease (Snyder et al. 1977). This is attributed to translocation of intestinal bacteria through the intestinal wall and portosystemic collaterals, and reduced immune response. The incidence of pericardial effusion in cirrhotic patients is approximately 32-63\% and correlates with the degree of liver failure (Shah and Variyam 1988). The effusion is usually small and may require drainage if it affects cardiac function. Patients with liver disease exhibit three common cardiac electrophysiological disturbances: electromechanical dissociation, prolongation of ventricular repolarization (the Q-T interval), and chronotropic incompetence (Milani et al. 2007).

Pulmonary hypertension associated with portal hypertension was first described in 1951 (Mantz and Craige 1951). Pulmonary hypertension defined as a mean pulmonary artery pressure of $>25 \mathrm{mmHg}$ and pulmonary vascular resistance of $>240 \mathrm{dyn} / \mathrm{s} / \mathrm{cm}^{-5}$ ( 3 Wood units) is more common in patients with liver disease, with a prevalence of $0.25-0.73 \%$ (Lebrec and Capron 1979; McDonnell et al. 1983). Pulmonary artery pressure is a function of pulmonary venous pressure, pulmonary vascular resistance, and cardiac output [(Pulmonary artery pressure $=$ Pulmonary venous pressure + (Pulmonary vascular resistance $\times$ Cardiac output)]. Therefore, pulmonary hypertension is not uncommon in patients with liver disease because of their poor left ventricular compliance, increased pulmonary vascular resistance, and increased pulmonary blood flow from portosystemic shunting. The pathophysiology and management of pulmonary hypertension are well-described in Chapter 9 - Hepatopulmonary Syndrome and Portopulmonary Hypertension.

Portal hypertension is caused by an increased intrahepatic vascular resistance and increased splanchnic blood flow. Endothelin-1, a powerful vasoconstrictor produced by the sinusoidal endothelial cells, is known to increase intrahepatic vascular resistance and activates stellate cells, and its level increases as cirrhosis progresses (Kojima et al. 2002; Gandhi et al. 1996). Normally, vasodilatory compounds, such as nitric oxide, counterbalance the increased intrahepatic vascular resistance induced by endothelin. In liver cirrhosis, however, nitric oxide production is inhibited by caveolin-1, a hepatic membrane protein that binds with endothelial nitric oxide synthase.

\section{Pulmonary System}

Hypoxemia of varying severity is present in 45-69\% of patients with significant liver disease (Krowka and Cortese 1985). The common causes are pleural effusions, impaired diffusion capacity, arteriovenous shunting, atelectasis caused by ascites or diaphragmatic dysfunction, aspiration secondary to encephalopathy, and deconditioning (Hourani et al. 1991). Ventilation-perfusion mismatch, pulmonary vasodilation, and infection also contribute to hypoxemia. Mild forms of hypoxemia are most common, although moderate-tosevere hypoxemia may be found in patients with advanced liver disease complicated by adult respiratory distress syndrome (ARDS), infection, and multiple organ failure. 
Hepatopulmonary syndrome, first described by Fluckiger in 1884 (Fluckiger 1884), may cause severe hypoxemia in a subset of patients with liver disease. The syndrome consists of a triad of liver dysfunction, severe hypoxemia $\left(\mathrm{PaO}_{2}<70\right.$ $\mathrm{mmHg}$ in room air), and pulmonary vasodilation, and is characterized by dyspnea, cyanosis, clubbing of the digits, exercise desaturation, and orthodeoxia (hypoxemia in upright position). Other concomitant clinical signs are a markedly increased alveolar-arterial oxygen gradient, portal hypertension, and vascular abnormality such as spider angioma and pulmonary vasodilation. The pulmonary vascular dilation (from $8-15 \mu$ to $15-100 \mu$ ) at the precapillary level is believed to be the main pathology of the hepatopulmonary syndrome, which is caused by decreasing erythrocyte transit time and impairing diffusion of oxygen to the erythrocytes at the center of the bloodstream (Genovesi et al. 1976). In contrast with other pulmonary diseases, oxygenation improves dramatically with a high inspired oxygen concentration $\left(\mathrm{FiO}_{2}\right)$, because a high alveolar concentration of oxygen overcomes the diffusion barrier and oxygenates the erythrocytes in the center of the bloodstream. The pathophysiology and management of hepatopulmonary syndrome are described in Chapter $9>$ Hepatopulmonary Syndrome and Portopulmonary Hypertension.

Non-cardiogenic pulmonary edema occurs in $37-79 \%$ of patients with advanced liver disease, particularly in those with fulminant hepatic failure, and appears to be associated with sepsis and a neurogenic mechanism. The presence of this complication is ominous: Matuschak and Shaw reported that all 29 patients who developed non-cardiogenic pulmonary edema died before liver transplantation (Matuschak and Shaw 1987). In contrast, a rapid reversal of ARDS after liver transplantation has been reported (Doyle et al. 1993). Pulmonary edema caused by fluid overload responds to diuretics and has a relatively benign course.

Pleural effusions are found on chest X-rays in about $10 \%$ of patients. These are caused by the unidirectional passage of ascites via diaphragmatic defects into the pleural space. Diagnostic thoracentesis is necessary to confirm the transudative nature and to exclude infection, malignancy, or embolic disease. Optimal control of ascites may prevent symptomatic pleural effusions, and transjugular intrahepatic portosystemic shunt (TIPS) is effective in treating refractory hydrothorax in $84 \%$ of patients (Siegerstetter et al. 2001).

\section{Renal System}

Approximately $10 \%$ of hospitalized cirrhotic patients with ascites develop the hepatorenal syndrome, which is a form of acute pre-renal kidney injury caused by circulatory dysfunction secondary to an imbalance between circulating vasodilatory and vasoconstrictive substances. The primary contributing factor for the hepatorenal syndrome is nitric oxide-induced vasodilation of the splanchnic vascular bed causing systemic arterial underfilling and relative hypovolemia (Arroyo et al. 1996). This relative hypovolemia activates baroreceptor-mediated sympathetic and the renin-angiotensin system to constrict all vascular beds including the renal vasculature (Guevara et al. 1998). The initial prostaglandin-mediated compensatory renal vasodilation is followed by renal arterial vasoconstriction and renal hypoperfusion. A striking feature of the hepatorenal syndrome is the lack of any histologic change and its reversibility: the affected kidneys resume their function after successful liver transplantation. The renal failure may be rapid (Type 1) or insidious (Type 2) and results in sodium and water retention and dilutional hyponatremia. Since the hepatorenal syndrome is a functional renal failure, the urine is similar to that found in pre-renal azotemia: oliguria, low urinary sodium, and an increased urine osmolality and urine to plasma osmolality ratio.

The major criteria for the diagnosis of the hepatorenal syndrome are as follows: (1) advanced hepatic disease and portal hypertension; (2) low glomerular filtration rate (serum creatinine $>1.5 \mathrm{mg} / \mathrm{dL}$ or creatinine clearance $<40 \mathrm{~mL} /$ min); (3) absence of nephrotoxic drug use, shock, systemic infection, or recent fluid losses; (4) lack of sustained improvement after diuretic 
withdrawal and volume resuscitation with $1.5 \mathrm{~L}$ of normal saline; (5) proteinuria ( $<500 \mathrm{mg} / \mathrm{dL})$; and (6) no ultrasound evidence of urinary obstruction or parenchymal disease. Minor criteria include oliguria $(<500 \mathrm{~mL} /$ day $)$, urinary sodium $<10 \mathrm{mEq} / \mathrm{L}$, urinary osmolality greater than plasma osmolality, urinary red blood cells (RBCs) $<50 / \mathrm{hpf}$, and serum sodium $<130 \mathrm{mEq} / \mathrm{L}$. It is noteworthy that conventional renal function tests, such as BUN and creatinine levels, overestimate renal function in patients with liver failure because malnutrition and muscle wasting contribute to a low creatinine level and liver dysfunction impairs urea synthesis.

The hepatorenal syndrome is treated with the administration of vasopressin-1 agonists (i.e., terlipressin), TIPS, and, most reliably, liver transplantation. One uncontrolled trial using terlipressin with albumin for a median duration of 26 days (range 8-68 days) showed improvement in serum sodium as well as a decrease in the creatinine level below $2 \mathrm{mg} / \mathrm{dL}$ (Mulkay et al. 2001). Hemodialysis is a temporary measure and its efficacy is not reliable. The only primary preventive measure showing some promise is the administration of albumin along with antibiotics as soon as the presence of spontaneous bacterial peritonitis is diagnosed; this possibly works by preventing hypovolemia and subsequent activation of vasoconstrictor systems.

\section{Coagulation System}

All phases of hemostasis are impaired in patients with liver disease, including clot formation, fibrinolysis, and their inhibitory processes. Thrombocytopenia is found in 30-64\% of cirrhotic patients, and platelet count is commonly below $75,000 / \mathrm{mm}^{3}$. Thrombocytopenia is primarily caused by splenomegaly associated with portal hypertension, which pools up to $90 \%$ of platelets in the spleen. However, the degree of thrombocytopenia does not closely correlate with the size of the spleen. Impaired hepatic synthesis of thrombopoietin also leads to thrombocytopenia. Thrombopoietin is involved in the maturation and formation of platelets, and its return to a normal level coincides with a gradual increase in platelet count by the fifth day after liver transplantation (Kawasaki et al. 1999). Other contributing factors are increased destruction of platelets by immune mechanisms, excessive activation of coagulation, and direct bone marrow suppression by toxins such as ethanol and folate deficiency. Additionally, platelet dysfunction is common, as demonstrated by impaired platelet aggregation to adenosine diphosphate (ADP), collagen, and thrombin (Rubin et al. 1979).

The liver produces all coagulation factors except for von Willebrand factor. Therefore, plasma levels of clotting factors are directly related to the severity of liver disease, and prothrombin time (PT) is considered to be one of the most sensitive hepatic synthetic function tests. The plasma fibrinogen level, being an acutephase reactant, typically is normal or increased in chronic liver disease. A reduction in the fibrinogen level may indicate either a greatly reduced hepatic reserve or significant extravascular loss to ascites. Markedly prolonged thrombin time indicates the presence of dysfibrinogenemia in some patients. Dysfibrinogenemia is characterized by an excessive number of sialic acid residues in the fibrinogen molecule and abnormal polymerization of fibrin monomers. Its clinical significance is unclear.

Patients with liver disease have a tendency to develop fibrinolysis due to decreased hepatic clearance of plasminogen activators, especially tissue plasminogen activator (tPA), and reduced production of $\alpha_{2}$-antiplasmin and thrombin activatable fibrinolysis inhibitors (Van Thiel et al. 2001). Elevated levels of D-dimers, fibrin degradation products, and plasminogen are present in ascitic fluid, indicating that absorption of ascitic fluid may contribute to the hyperfibrinolysis.

On the other hand, excessive activation of coagulation is common in liver disease because of inadequate hepatic clearance of activated coagulation factors, reduced level of coagulation inhibitors, and enlarged vascular beds. The hypercoagulable state may lead to localized or disseminated intravascular coagulation (DIC), particularly in the presence of sepsis, trauma, or 
major surgery. The diagnosis of excessive activation of coagulation is based on the presence of a known triggering factor and the progressively worsening of coagulation with thrombocytopenia.

\section{Anesthesia Consultation}

An anesthesia consultation is performed once a patient with ESLD is referred to the liver transplantation center. The type of liver disease is identified because patients with hepatocellular disease may have more pronounced hepatic dysfunction than those with cholestatic disease or hepatocellular cancer, and certain types of liver disease may affect other vital organ function (i.e., hemochromatosis, familiar amyloidosis, etc.). The anesthesia consultation is focused on evaluation of the functional reserve of extrahepatic organs, and various tests or specific consultations may be requested (Table 2).

\section{Cardiovascular Assessment}

Cardiovascular assessment is performed to determine two things: (1) whether a patient can be expected to survive the operation and immediate postoperative period; and (2) whether transplantation in patients with severe cardiopulmonary disease would be futile and an inappropriate use of a scarce donor organ (Lentine et al. 2012). A suggested strategy for cardiac assessment is shown in Fig. 3 (Raval et al. 2011). Overall cardiac performance is evaluated by transthoracic echocardiography to assess myocardial contractility, abnormality in cardiac anatomy, intracardiac or intrapulmonary shunting, and pulmonary artery pressure. Most patients over age 50 years undergo non-invasive stress testing because they may have multiple CAD risk factors (i.e., diabetes, hypertension, hyperlipidemia, and pre-existing cardiovascular disease), and limited physical activity masks underlying ischemic heart disease. An exercise stress test may not be feasible in many patients with advanced liver disease, and dobutamine stress echocardiography (DSE) is commonly used, although adenosine or dipyridamole may be used when dobutamineinduced tachycardia is not desirable. DSE, with its high sensitivity and specificity, appears to be the most reliable screening test (Plotkin et al. 1998), and dobutamine-induced tachycardia may mimic intraoperative stress on the cardiovascular system. On the contrary, DSE has been reported to have poor sensitivity (as low as $13 \%$ ) and negative predictive value (as low as $75 \%$ ) (Harinstein et al. 2008), and its results may not correlate with adverse cardiac events within 30 days after transplantation (Safadi et al. 2009).

Cardiac CT scan is a non-invasive technique measuring calcium deposits within the coronary vasculature. The total amount of calcium, adjusted to the age and gender of the patient, is reported as a calcium score. High scores suggest a greater potential for coronary artery stenosis (Shaw et al. 2003; O'Rourke et al. 2000), and a calcium score of $>400$ has a predictive value of cardiac complications within 1 month after transplantation (Kemmer et al. 2014). This test, however, may have limited predictive value as a single screening study for CAD. Cardiac CT angiography is an alternative to invasive coronary angiography. It does appear to have negative predicting value of $100 \%$ for clinical coronary events in patients undergoing liver transplantation (Cassagneau et al. 2012) but may not be suitable for the diagnosis of obstructive lesions at this time.

Because of the difficulty in diagnosing CAD using non-invasive testing methods, coronary angiography is recommended for patients with a positive DSE or multiple high-risk factors to identify the degree and type of obstruction. In addition, coronary angiography should be able to detect non-obstructive lesions (coronary artery stenosis $<50 \%$ ), which are unlikely to be detected by stress tests but can be responsible for acute coronary syndromes (unstable angina, myocardial infarction, or sudden cardiac death) (Rubin et al. 1994; Gulati et al. 2009). Cardiac catheterization, however, can be difficult in patients with severe liver disease due to bleeding complications and the increased risk of contrast-induced nephropathy (Sharma et al. 2009). 
Table 2 Liver transplantation evaluation at the Thomas Jefferson University Hospital

\begin{tabular}{lc}
\hline Jefferson Transplant Institute & David Sass, MD $\bullet$ Steven K Herrine MD $\bullet$ \\
& Jesse Civan, MD $\bullet$ Dina Halegoua, MD $\bullet$ Jonathan Fenkel, MD $\bullet$ \\
& Manish Thapar, MD $\bullet$ Scott Fink, MD $\bullet$ Victor Araya, MD \\
Liver Transplant Evaluation & Phone: $215-955-8900$ Fax: $215-503-2626$ \\
Lab/Diagnostic Testing Request & email: liver.evals@lists.jefferson.edu
\end{tabular}

\begin{tabular}{|c|c|}
\hline \multirow[b]{2}{*}{ Patient Name } & \multirow{3}{*}{$\begin{array}{l}\text { Patient DOB } \\
\text { Referring Gastroenterologist }\end{array}$} \\
\hline & \\
\hline Hepatologist & \\
\hline \multirow{2}{*}{$\frac{\text { Laboratory }}{\text { Chemistry } / \text { Hematology }}$} & Diagnostic Testing \\
\hline & $D *$ PPD with Anergy Panel \\
\hline CBC with diff and platelets & $0 *$ Chest $\mathrm{X}$ ray, $\mathrm{PA}$ and Lateral \\
\hline PT/PTT/INR & $\square *$ Upper GI endoscopy \\
\hline \multirow{2}{*}{ Comprehensive metabolic profile } & $\square$ Colonoscopy (age/risk factor appropriate) \\
\hline & $\square *$ Mammography (women over 40) \\
\hline $\begin{array}{l}\text { Fe, TIBC, Ferritin } \\
\text { Alpha-Fetoprotien (tumor marker) }\end{array}$ & $\square$ Pap Smear \\
\hline Serum Protein Electrophoresis & Hepatobiliary Imaging \\
\hline TSH and Free T4 & $\square$ MRI with gadolinium \\
\hline UA (urinalysis ) & $\square \mathrm{CT}$ of abdomen \& pelvis with contrast \\
\hline \multirow{2}{*}{$\begin{array}{l}\text { Alpha } 1 \text { anti-trypsin level with phenotype } \\
\text { Ceruloplasmin }\end{array}$} & GUS with Doppler \\
\hline & Cardiac Testing \\
\hline *PSA (men over 40) & $\square$ Echocardiogram with Doppler and Bubble Study \\
\hline $\begin{array}{l}\text { * Blood Type }(\mathrm{ABO}) \\
\text { Virology/Micro }\end{array}$ & $\square 12$ Lead EKG \\
\hline Virology/Micro & Pulmonary Studies \\
\hline $7^{*} \mathrm{HIV}$ & $\square \mathrm{ABG}$ on $100 \% \mathrm{O} 2$ \\
\hline *HCV Antibody & Vaccinations \\
\hline - $\mathrm{HBsAg}, \mathrm{HBsAb}, \mathrm{HBcAb}$ & $\square$ Hepatitis B \\
\hline$\beth * \mathrm{HAV} \lg \mathrm{G}+\mathrm{IgM}$ & Hepatitis A \\
\hline${ }^{*} \mathrm{CMV}, \mathrm{EBV}, \mathrm{VZV} \mathrm{Ab}$ & DPneumovax \\
\hline FRR & Consultations \\
\hline \multirow{2}{*}{$\begin{array}{l}\text { Quantiferon Gold } \\
\text { Immunology }\end{array}$} & $\square$ Anesthesiology (transplant) \\
\hline & $\square$ Social Work \\
\hline DANA & Gransplant Surgery \\
\hline \multirow{2}{*}{$\begin{array}{l}\text { ISMA } \\
\text { AMA }\end{array}$} & GDental \\
\hline & \\
\hline \multicolumn{2}{|l|}{ Tests below to be done only if clinically indicated } \\
\hline Laboratory & Diagnostic Testing \\
\hline Drug \& Alcohol Screen & Dobutamine Stress Echo (preferred; beta blockers must be held for \\
\hline HCV Quantitative RNA by PCR & $48 \mathrm{hrs}$ before) \\
\hline HCV Genotype & $\square$ Adenosine Stress test (Wt. $<175 \mathrm{lbs})$ \\
\hline HBV DNA, HbeAg, HbeAb & $\square$ SESTA MIBI (Wt > $175 \mathrm{lbs}$; large ascites) \\
\hline בHDV $\mathrm{Ab}$ & Full Pulmonary Function Tests, (age > 50 and /or smoking hx) \\
\hline HFE (genetic testing for Hemochromatosis) & Carotid study/Cerebrovascular Duplex (Age $>50$ with h/o vascular \\
\hline HLA A, B, C, DR \& PRA (for liver/kidney patients only) & disease ) \\
\hline Jother: & $\square$ Chest CT scan (for HCC patients) \\
\hline Consultations & $\square$ DEXA scan if clinically indicated \\
\hline Pulmonary & DERCP \\
\hline Cardiology & Liver Biopsy \\
\hline Psychiatry & $\square$ Percutaneous, by Hepatologist \\
\hline Substance Abuse Evaluation & $\square$ Ultrasound Guided, by Radiology \\
\hline Rehab Medicine & $\square$ Transjugular, by CVIR \\
\hline Jephrology & Vaccinations \\
\hline EEndocrinology & $\square$ Meningococcal \\
\hline Jinfectious Disease & $\square$ Hemophilus influenza B \\
\hline Jutrition & $\square^{\text {Seasonal influenza }}$ \\
\hline Indicates test/consults acceptable within 12 months of & MELD: \\
\hline evaluation & MELD-Na \\
\hline & Expedite: $\square$ YES \\
\hline & Indication for Expedited: \\
\hline
\end{tabular}

If significant coronary artery stenosis $(>70 \%$ stenosis) is detected, revascularization may be attempted before liver transplantation. Bare metal stents are favored over drug-eluting stents to avoid the need for long-term antiplatelet therapy (6 weeks vs. 1 year). When angioplasty is not amenable, coronary artery bypass grafting (CABG) is performed. It is clear that 1-year survival after CABG is greater in patients with Child-Pugh Class A $(80 \%)$ than with Child-Pugh Class B 


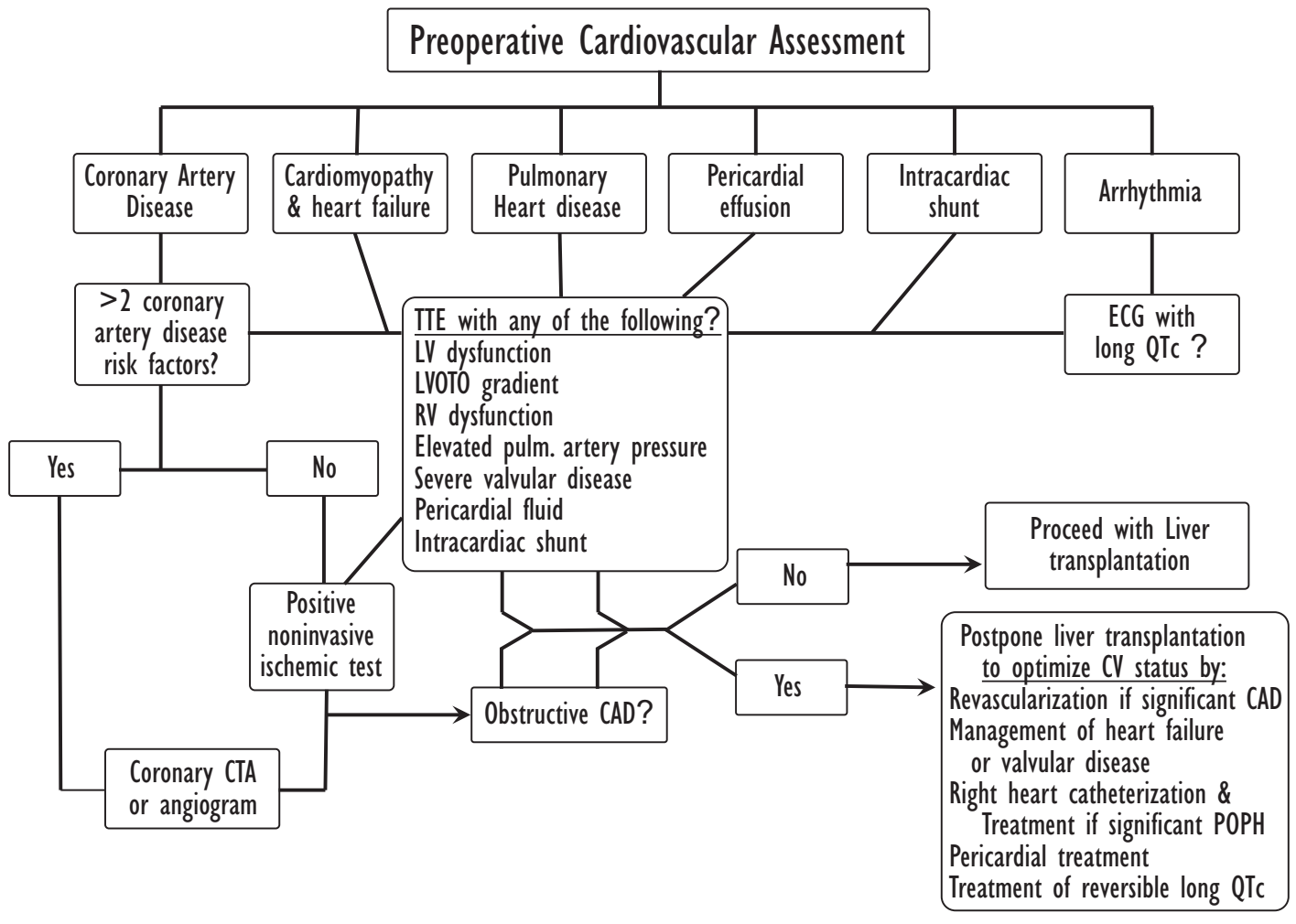

Fig. 3 Suggested strategy for preoperative cardiac assessment of liver transplantation candidates (Reprinted from JACC, 58, Raval Z, Harinstein ME, Skaro AI et al.,

(45\%) and C (16\%) (Filsoufi et al. 2007). Therefore, patients with Child-Pugh Class A can undergo CABG relatively safely while waiting for liver transplantation. On the other hand, patients with Child-Pugh Class B and C may require simultaneous $\mathrm{CABG}$ and liver transplantation.

Patients with mild-to-moderate valvular disease undergo liver transplantation without excessive complications. Similar to that of $\mathrm{CABG}$, mortality after corrective valvular surgery depends on the severity of liver disease. Therefore, Child-Pugh Class $\mathrm{C}$ patients with severe aortic or mitral valve stenosis may undergo percutaneous balloon valvuloplasty or simultaneous valve replacement with cardiopulmonary bypass and liver transplantation.

Myocardial disease is commonly detected by transesophageal echocardiography (TEE). Patients with chronic cardiomyopathy may have attenuated systolic contraction and diastolic relaxation, altered repolarization, and reduced cardiac
Cardiovascular risk assessment of the liver transplant candidate, 223-231, Copyright (2011), with permission from Elsevier)

response to $\beta$ stimulation (Liu et al. 2002). Patients with moderate-to-severe cardiomyopathy may be excluded from candidacy. Moderate-tosevere hypertrophic obstructive cardiomyopathy (HOCM) can cause left ventricular outflow obstruction by systolic anterior motion (SAM) of the anterior leaflet of mitral valve, especially in the presence of tachycardia, hypovolemia, and $\beta$ stimulation. In these patients, preload and afterload should be optimized under the guidance of TEE, and tachycardia and $\beta$ stimulation should be avoided. Surgical correction of the condition should be considered before or at the time of liver transplantation.

\section{Pulmonary Evaluation}

For pulmonary evaluation, results of chest X-ray, arterial blood oxygen tension in $100 \%$ oxygen, and spirometry are reviewed to identify the 
degree of pulmonary shunting, obstructive, or restrictive disease. When the hepatopulmonary syndrome is suspected, contrast TEE or TC-99 m macro aggregated albumin scintigraphy may be performed for its definitive diagnosis (Krowka et al. 2000).

\section{Renal Function}

For evaluation of renal function, results of BUN, creatinine, glomerular filtration rate, levels of serum and urine electrolytes, urine output, and renal ultrasound are reviewed. The diagnosis criteria of the hepatorenal syndrome have been described earlier. In patients with chronic renal failure, simultaneous liver and kidney transplantation is performed, the criteria for which are end-stage renal disease with dialysis, no dialysis but a glomerular filtration rate $<30 \mathrm{~mL} / \mathrm{min}$ and proteinuria $>3 \mathrm{~g}$ /day with a 24 -h urine protein/ creatinine ratio $>3$, and acute kidney injury requiring dialysis at least twice per week for more than 6 weeks (Charlton et al. 2009).

\section{Fulminant Hepatic Failure}

In patients with fulminant hepatic failure, reversibility of the neurologic function should be investigated using clinical signs, EEG, brain CT scan, the cerebral metabolic rate of oxygen, and TCD. In addition, ICP monitoring is recommended when a high ICP $(>25 \mathrm{mmHg})$ is suspected, although its benefit should be weighed against potential complications (Vaquero et al. 2005). Poor prognostic indicators of fulminant hepatic failure are progressive hepatic failure for 7-14 days, grade 3-4 encephalopathy, intracranial hypertension, cerebral swelling, severe coagulopathy, rapid shrinkage of the liver, metabolic acidosis, hemodynamic instability, and sepsis.

\section{Coagulation System}

For the coagulation system, PT, activated partial thromboplastin time (aPTT), and platelet count are reviewed. In general, no specific coagulation therapy is requested because of the potential long waiting period and fluid overloading. Abdominal magnetic resonance imaging (MRI) is reviewed to assess the degree of portosystemic shunting and anatomy of hepatic vasculature. Additional consultation may be requested from various specialists to identify the type and severity of the specific organ dysfunction.

\section{Contraindications}

After the evaluation, all information of the potential recipient is compiled to stratify whether the patient's condition can be optimized or meet the criteria of contraindications. Contraindications are diseases or conditions patients could have that may not improve survival after liver transplantation. They include malignancy with poor prognosis, active bacterial and viral infection, severe cardiopulmonary dysfunction, and technical difficulties. Active alcoholism is a contraindication, although demonstrable abstinence for 6 months is considered acceptable. The presence of multiple organ dysfunction is a relative contraindication for liver transplantation as the 2-year survival is approximately $25 \%$. Indications and contraindications of liver transplantation, however, have evolved over the past 50 years, and further modifications are expected to occur.

\section{Candidate Selection}

Anesthesiologists participate in the Transplantation Candidate Selection Committee for discussion of the hepatic disease, its complications, and the extrahepatic organ function of each patient. Once the patient is placed on the active candidate list, the United Network for Organ Sharing (UNOS) is notified and the patient is given a MELD (Model for End-Stage Liver Disease) or PELD (Pediatric End-Stage Liver Disease) score for fair distribution of donor livers. 


\section{Surgical Aspects of Liver Transplantation}

Although surgical techniques are fully described elsewhere, a brief description of their physiologic effects is warranted here.

In OLT, after removal of the diseased liver, the donor liver is placed anatomically in the right upper quadrant. For the convenience of description, the procedure is divided into three stages: stage 1 (dissection stage), stage 2 (anhepatic stage), and stage 3 (neohepatic stage). The dissection stage begins with an inverted Y-shaped bilateral subcostal skin incision and ends with the skeletonization of the diseased liver. The anhepatic stage begins with the occlusion of the hepatic artery, portal vein, and IVC for hepatectomy. However, the patient is virtually anhepatic once the hepatic artery or portal vein is occluded. Three surgical techniques are used for hepatectomy and vascular reconstruction during the anhepatic stage: OLT with simple venous crossclamping, OLT with venovenous bypass, and the piggyback technique.

\section{Orthotopic Liver Transplantation (OLT) with Simple Venous Cross-Clamping}

In OLT with simple venous cross-clamping, the diseased liver is removed together with the retrohepatic portion of the IVC after crossclamping of the suprahepatic and infrahepatic IVC, hepatic artery, and portal vein (Fig. 4). After surgical hemostasis of the hepatic bed, the donor liver is placed in the right upper quadrant, and sequential anastomoses of the suprahepatic IVCs, infrahepatic IVCs, portal veins, and hepatic arteries are performed. During the infrahepatic IVC anastomosis, the liver allograft is flushed with $1000 \mathrm{~mL}$ of cold lactated Ringer's solution or $5 \%$ albumin solution through a cannula in the portal vein. This flush technique allows preservation solution, metabolites, and air in the donor liver to escape through the incompletely anastomosed infrahepatic IVC. A second flush may be used by allowing $300-500 \mathrm{~mL}$ of blood to escape through the incompletely anastomosed portal vein by unclamping the infrahepatic IVC (back-bleeding technique). When the portal vein of the recipient is less than optimal, the superior mesenteric vein, collateral vein, or venous graft may be used for portal blood supply. The hepatic artery is reconstructed by end-to-end hepatic arterial anastomosis. However, an arterial graft is placed between the graft hepatic artery and the infrarenal aorta of the recipient with a side clamp on the aorta when the size or anatomy of the recipient hepatic artery is less than optimal.

The liver is reperfused by the sequential unclamping of the infrahepatic IVC, portal vein, suprahepatic IVC, and hepatic artery. After hemostasis, choledochocholedochostomy is performed frequently with a T-tube. Choledochojejunostomy using a Roux-en-Y loop is performed when the bile ducts are diseased or mismatched in size. The abdomen is closed once the absence of foreign bodies in the peritoneal cavity is confirmed. In patients with a large graft or swollen intestine, the abdomen may require secondary closure.

\section{OLT with Venovenous Bypass}

OLT with venovenous bypass was developed in 1983 to minimize reduction of venous return associated with the cross-clamping of the IVC and portal vein by diverting blood from the IVC and portal vein to the axillary vein using a centripetal magnetic pump (Shaw et al. 1984). Once the hepatic hilum is dissected, cannulas are inserted into the left superficial femoral vein $(7 \mathrm{~mm})$ and portal vein $(9 \mathrm{~mm})$ for outflow from the patient and into the left axillary vein $(7 \mathrm{~mm})$ for venous inflow. The cannula site and size may vary depending on the preference of the surgical team or anatomic variations. The cannulas and heparin-bonded tubings are flushed with heparin solution (2000 U/L) to avoid thrombosis during preparation. Systemic heparinization is not used because of the presence of pre-existing coagulopathy and the use of heparin-bonded tubings. The bypass run begins by unclamping all cannulas while the pump speed is gradually increased to achieve the maximal flow rate. Hepatectomy and anastomoses of the 

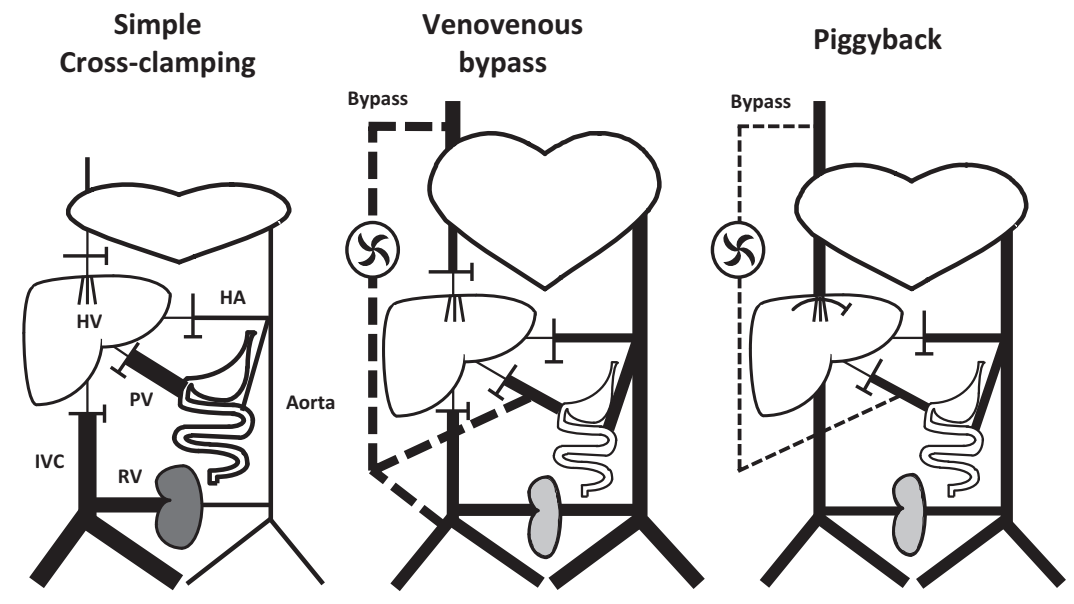

Fig. 4 Schematic diagram of three surgical techniques during the anhepatic stage. Line thickness indicates relative blood flow to each vasculature. The simple cross-clamping technique reduces venous return and cardiac output and leads to congestion of the viscera, kidneys, and lower

suprahepatic and infrahepatic IVC are performed once full bypass is achieved. The removal of the portal cannula for portal venous anastomosis leads to a partial bypass, which reduces venous return. Bypass is terminated after the engrafted liver is reperfused, and cannulas are removed.

The advantages of venovenous bypass are (1) well-preserved cardiac output by uninterrupted venous return from the viscera and lower extremities; (2) effective decompression of the portal venous system, which decreases bleeding and intestinal congestion; (3) avoidance of renal congestion, oliguria, and hematuria; and (4) simplified anhepatic stage allowing meticulous hepatectomy and vascular anastomoses. Long-term complications are neurovascular injury, thrombosis, infection, lymphocele, and seroma at the cannulation sites.

As an alternative to the traditional venovenous bypass technique, percutaneous cannulation was introduced. In this technique, inflow to the patient is achieved by percutaneous cannulation of the right internal jugular vein (16-20 French) performed by the anesthesia team using a Seldinger technique, and outflow from the patient by percutaneous cannulation of the left femoral vein (16-20 French) and a portal cannula by the surgical team. This technique is generally safe, but extremities. The venovenous bypass technique maintains venous return without visceral congestion. Hemodynamic changes in the piggyback technique are between the two other techniques. $H A$ hepatic artery, $H V$ hepatic vein, $I V C$ inferior vena cava, $P V$ portal vein, $R V$ renal vein)

the inadvertent extravascular placement of an inflow cannula may cause a massive hemothorax (Sakai et al. 2007).

\section{Piggyback Technique}

The piggyback technique was originally designed for patients with significant cardiovascular disease, portacaval shunt, superior vena caval syndrome, or small donor livers (Tzakis et al. 1989). In this technique, the diseased liver is removed without the retrohepatic portion of the IVC by peeling the diseased liver off the IVC after transaction of the hepatic veins, hepatic artery, and portal vein. Therefore, systemic venous return can be relatively well preserved via the intact IVC during the anhepatic stage. Vascular anastomoses are made between the reconstructed ostia of the recipient by combining hepatic veins and the suprahepatic IVC of the graft for the drainage of the hepatic venous blood. The portal vein of the recipient and the graft are anastomosed for portal blood supply, and the infrahepatic IVC of the graft is ligated.

The neohepatic stage begins with reperfusion of the grafted liver by sequential unclamping of the infrahepatic IVC, portal vein, suprahepatic IVC, and hepatic artery, although the sequence of unclamping 
may vary depending on the surgical technique. Reperfusion is followed by hepatic arterial anastomosis (if it has not been performed already), biliary reconstruction, and closure of the abdomen.

\section{Preparation and Anesthetics}

Immediate preoperative consultation is made when a donor organ is identified. The patient is re-evaluated to identify any interval changes during the waiting period. Anesthetic and postoperative management and their risks are explained to the patient one more time. In general, pre-medication is withheld in most cases because of potential encephalopathy and hypovolemia, and narcotics (e.g., fentanyl $1-5 \mu \mathrm{g} / \mathrm{kg}$ ) are commonly administered intravenously in the operating room.

Necessary medications and anesthesia equipment are listed in Table 3. A device that delivers fluids and blood rapidly on demand is considered standard equipment (i.e., FMS2000 ${ }^{\circledR}$ fluid warming system, Belmont Instrument Corp., Billerica, MA, USA) (Elia and Kang 2002). An autotransfusion system is helpful in minimizing the need for bank blood (Dzik and Jenkins 1985; Kang et al. 1991). A system that monitors coagulation, either a conventional coagulation profile, Thromboelastography $\mathrm{R}$ with circle (TEG; Haemonetics, Braintree, MA, USA), or Rotational Thromboelastometry $\mathrm{R}$ with circle (ROTEM; TEM International, Basel, Switzerland), is essential in monitoring and management of coagulation (Kang 1986, 1997). TEG and ROTEM provide similar physical properties of blood coagulation, although TEG monitors shear elasticity and ROTEM monitors viscoelasticity. In general, 20 units each of cross-matched packed RBCs (PRBCs) and fresh frozen plasma (FFP) are available at all times, and 10 units of each are prepared in the operating room. Platelets (10-20 units) should be available on demand.

Two large-bore intravenous (IV) catheters (up to 8.5 or 9 French) are secured, typically in the right antecubital and right or left internal jugular vein. When the antecubital vein is unavailable, two catheters may be placed in the same internal jugular vein. Catheter patency is
Table 3 Anesthesia equipment and medications for liver transplantation

\begin{tabular}{|c|}
\hline Equipment \\
\hline Anesthesia machine with volume ventilator \\
\hline Mass spectrometer or capnograph \\
\hline Multiple-channel vital-sign monitor \\
\hline Pulse oximeter \\
\hline $\begin{array}{l}\text { Cardia output computer (oximetry or right ventricular } \\
\text { ejection fraction) }\end{array}$ \\
\hline Transesophageal echocardiograph \\
\hline Drug infusion pumps \\
\hline Thromboelastograph or thromboelastometer \\
\hline Rapid-infusion device \\
\hline Autotransfusion system \\
\hline Warming blanket \\
\hline Defibrillator \\
\hline Medications \\
\hline Induction agents \\
\hline Propofol \\
\hline Etomidate \\
\hline Intravenous agents \\
\hline Midazolam \\
\hline Fentanyl \\
\hline Inhalation agents \\
\hline Isoflurane \\
\hline Muscle relaxants \\
\hline Succinylcholine \\
\hline Rocuronium \\
\hline Vecuronium \\
\hline Other drugs \\
\hline Atropine $(0.4 \mathrm{mg})$ \\
\hline Calcium chloride $(100 \mathrm{mg} / \mathrm{mL}, 200 \mathrm{~mL})$ \\
\hline $\mathrm{NaHCO}_{3}(40 \mathrm{mmol} / 50 \mathrm{~mL}, 400 \mathrm{mmol})$ \\
\hline Tromethamine (THAM) (500 mL, $1500 \mathrm{~mL})$ \\
\hline Ephedrine (5 mg/mL, 20 mL) \\
\hline Epinephrine $(4 \mu \mathrm{g} / \mathrm{mL}, 20 \mathrm{~mL})$ \\
\hline Epinephrine $(40 \mu \mathrm{g} / \mathrm{mL}, 20 \mathrm{~mL})$ \\
\hline$\varepsilon$-Aminocaproic acid (EACA) $(250 \mathrm{mg} / \mathrm{mL}, 10 \mathrm{~mL})$ \\
\hline Protamine $(10 \mathrm{mg} / \mathrm{mL}, 10 \mathrm{~mL})$ \\
\hline Insulin: available in the refrigerator \\
\hline Potent vasoactive drips: as needed \\
\hline
\end{tabular}

confirmed by noting the line infusion pressure of $<300 \mathrm{mmHg}$ during fluid infusion at $500 \mathrm{~mL} /$ min. Sterile technique should be followed during catheterization, and antiseptic ointment or antiseptic patch is applied at the skin puncture site. A nasogastric tube is placed with copious lubrication and topical vasoconstrictor to avoid nasal or esophageal variceal bleeding. 
Proper monitoring is prerequisite to a successful outcome because patients undergoing liver transplantation develop clinically significant hemodynamic, hematologic, metabolic, and other homeostatic abnormalities. Non-invasive monitoring is similar to that for patients undergoing any major surgery. For invasive monitoring, two intraarterial catheters (20 gauge in the left radial artery and 16-18 gauge in the right femoral artery) are used at the Thomas Jefferson University Hospital. Femoral arterial pressure monitoring is preferred because it reflects central arterial blood pressure more accurately in the presence of low systemic vascular resistance, particularly after reperfusion (Lee et al. 2015). Radial arterial pressure monitoring is useful for blood sampling and backup pressure monitoring when the aorta is partially or completely clamped during aorta-to-hepatic artery anastomosis. A pulmonary artery catheter (PA catheter) is inserted via the right internal jugular vein to monitor cardiac output, intracardiac pressures, and core temperature. Carotid artery puncture should be assiduously avoided because of the presence of coagulopathy. An oximetric-type PA catheter provides additional information on mixed venous hemoglobin oxygen saturation $\left(\mathrm{SvO}_{2}\right)$. The right ventricular ejection fractiontype PA catheter monitors the right ventricular ejection fraction and right ventricular end-diastolic volume. It has been shown that central venous pressure (CVP) and pulmonary capillary wedge pressure (PCWP) are not as sensitive as right ventricular end-diastolic volume in estimating preload, particularly during the anhepatic stage (DeWolf et al. 1993b). Recently, non-invasive, continuous cardiac output monitoring was introduced; however, the technique is not reliable in monitoring cardiac output in hyperdynamic patients. In some centers, a CVP catheter is used instead of a PA catheter. This, of course, is justified if hemodynamic derangement is kept minimal during the entire surgical procedure. However, most centers use a PA catheter for three reasons: (1) hemodynamic instability can be unpredictable during liver transplantation; (2) determination of cardiac output and preload is more clinically significant than CVP monitoring; and (3) it is an important educational tool for trainees. TEE is used in all patients at the Thomas Jefferson University Hospital to monitor myocardial contractility, ventricular end-diastolic volume, wall motion abnormality, air or thromboembolism, intrapulmonary shunting, and patency of the reconstructed major veins. A TEE probe may cause esophageal variceal bleeding (Burger-Klepp et al. 2012) and it should therefore be placed gently.

Various laboratory tests are performed, including arterial blood gas tension and acid-base state, and serum level of electrolytes, ionized calcium, glucose, lactate, and ionized magnesium if available. Typical test times are before and after induction of anesthesia, every hour during the dissection stage, $5 \mathrm{~min}$ after the onset of the anhepatic stage, every $30 \mathrm{~min}$ during the anhepatic stage, $15 \mathrm{~min}$ before reperfusion, 5 and $30 \mathrm{~min}$ after reperfusion, and every hour thereafter. Coagulation is monitored by conventional coagulation profile (PT, aPTT, fibrinogen level, and platelet count) and TEG or ROTEM at the following times: before induction of anesthesia, every hour during the dissection stage, 15 and $60 \mathrm{~min}$ after onset of the anhepatic stage, $15 \mathrm{~min}$ before reperfusion, 5 and 30 min after reperfusion, and every hour thereafter.

Monitoring of TEG or ROTEM and the platelet count is preferable as a conventional coagulation profile has several drawbacks when used during liver transplantation (Kang 1995). PT is a very sensitive hepatic function test and is prolonged in most patients undergoing liver transplantation. Administration of FFP to correct the PT may not be possible or desirable in the course of surgery. aPTT follows a similar time course to PT, and its correction may not be practical. It is a sensitive test for the heparin effect, and its prolongation indicates the presence of heparin released from the bypass circuit or grafted liver. The fibrinogen level is frequently maintained within the acceptable range, although severe hypofibrinogenemia may indicate either active fibrinolysis or excessive activation of coagulation. The level of fibrin(ogen) degradation products is usually elevated in most patients due to excessive activation of coagulation 
Fig. 5 Effects of pharmacologic agents on pathologic coagulation immediately after reperfusion (From Kang YG (1986) Monitoring and treatment of coagulation. In: Winter PM, Kang YG (ed) Hepatic

Transplantation, anesthetic and perioperative management. Prager, New York, with the permission of the publisher)

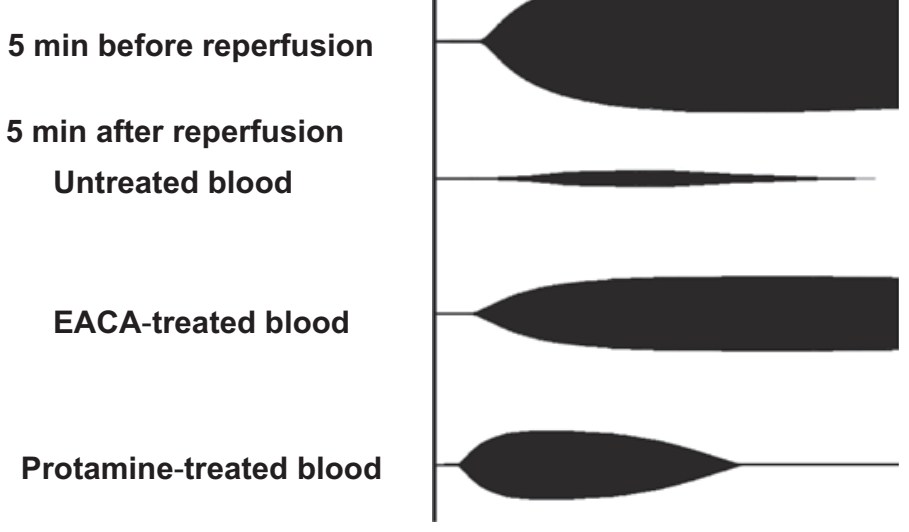

and reabsorption of defibrinated blood from the abdominal cavity and does not have any immediate clinical significance. Further, coagulation profile results may not be available in a timely manner.

TEG/ROTEM has several advantages over a conventional coagulation profile and has been accepted as a standard coagulation monitoring tool by the ASA (American Society of Anesthesiologists 2015). It rapidly and reliably measures blood coagulability (quality) instead of the quantity of each coagulation component. An accurate differential diagnosis can be made for replacement therapy and pharmacologic therapy by comparing TEG/ROTEM of untreated blood with that of blood treated with various blood components (FFP, platelets, cryoprecipitate) or pharmacologic agents (protamine sulfate, heparinase, $\varepsilon$-aminocaproic acid [EACA], aprotinin) (Fig. 5) (Kang et al. 1987).

Lastly, circumferential identification tags around the wrists or ankles are removed to avoid the compartment syndrome. Both arms are placed on padded arm boards in an abducted position, and excessive abduction should be avoided to prevent a plexus stretch injury. The extremities are protected with foam padding to avoid pressure injuries.

A rapid-sequence induction is preferred because of uncertain gastric emptying. Anesthesia is commonly induced with propofol $(2-3 \mathrm{mg} / \mathrm{kg})$ or etomidate $(0.3 \mathrm{mg} / \mathrm{kg})$, and fentanyl $(2-5 \mu \mathrm{g} / \mathrm{kg})$ is frequently added. Succinylcholine $(1-2 \mathrm{mg} / \mathrm{kg})$ or rocuronium bromide $(1.2 \mathrm{mg} / \mathrm{kg})$ is used to facilitate intratracheal intubation. Anesthesia is maintained using volatile inhalation agents and narcotics. Isoflurane is the preferred inhalation agent because its effect includes less myocardial depression and biotransformation. Nitrous oxide is avoided because it distends the bowel and increases the size of any entrained air. Midazolam (1-4 mg) may be added for amnesia. For muscle relaxation, rocuronium bromide, vecuronium bromide, or cisatracurium besilate are commonly used.

Antibiotics and immunosuppressants administered during surgery may vary from center to center. At the Thomas Jefferson University Hospital, Unasyn $^{\circledR}$ (ampicillin/sulbactam $3 \mathrm{~g}$ ) is given before incision and every $4 \mathrm{~h}$ thereafter. For patients allergic to cephalosporin or penicillin, vancomycin is administered within $2 \mathrm{~h}$ before skin incision $(1 \mathrm{~g}$ for patients $<80 \mathrm{~kg}$ and $1.5 \mathrm{~g}$ for those $>80 \mathrm{~kg}$ ). For immunosuppression, methylprednisolone (500 $\mathrm{mg}$ IV) and basiliximab (20 mg IV) are given during the anhepatic stage and tacrolimus is given in the postoperative period.

\section{Physiologic Homeostasis During Liver Transplantation}

Liver transplantation imposes a great deal of physiologic stress on patients, and maintenance of physiologic homeostasis is essential to a successful outcome. 


\section{Cardiovascular Homeostasis}

The goal of hemodynamic management is to optimize tissue perfusion by maintaining the hyperdynamic state characteristic of ESLD. In general, there are two schools of thought about maintaining hemodynamic stability. The first endorses maintaining the hyperdynamic state to optimize cardiac output and tissue perfusion. Patients with ESLD have generalized vasodilation and are known to have oxygen debt at the tissue level. Therefore, maintaining the hyperdynamic state, instead of 'normal blood pressure,' ensures ample oxygen delivery to tissues and avoids tissue acidosis. This, in turn, optimizes tissue metabolism and hepatic blood flow. The second school of thought endorses maintaining arterial blood pressure within the normal range. This may include the use of various vasopressors (phenylephrine, norepinephrine, vasopressin, etc.) with or without hypovolemia. In extreme cases, blood is removed from the patient to induce hypovolemia, and blood pressure is supported by vasopressors (Massicotte et al. 2010); it has been claimed that blood loss is minimal without increasing perioperative complications, although fluid restriction may lead to tissue ischemia, renal failure, and air embolism (Melendez et al. 1998; Schroeder et al. 2004). Further, $\alpha$-vasopressors (norepinephrine and phenylephrine) decrease hepatic blood flow by reducing portal venous flow dramatically in the presence of a limited hepatic arterial buffer response (Mehrabi et al. 2005).

\section{Dissection Stage}

Hemodynamic instability represented by reduced cardiac output and hypotension is typically caused by hypovolemia associated with drainage of ascites, rapid third-space fluid loss, surgical bleeding, and inadvertent compression of major vessels (IVC, portal vein, hepatic veins, and aorta). Intravascular volume is usually replenished by administration of a mixture of PRBCs and FFP (typically, PRBC:FFP: PlasmaLyte- $A^{\circledR}=200: 300: 250 \mathrm{~mL}$ ) using a rapid-infusion device. This mixture yields hematocrit of $26-28 \mathrm{vol} \%$ and coagulation factor levels of $30-50 \%$ of normal. A low hematocrit is chosen to optimize microcirculation and minimize the RBC wastage. Calciumcontaining fluid (i.e., lactated Ringer's solution) should not be used to prevent clot formation in the reservoir of the rapid-infusion device. Continuous administration of FFP is necessary to compensate for the loss of coagulation elements (procoagulants, prolysins, and their inhibitors) by surgical bleeding and excessive activation of coagulation. In patients with minimal blood loss, colloids (albumin or FFP) and crystalloids may be required to compensate for the third-space fluid loss and continuous production of ascites. Close communication with the surgical team is essential to identify the cause of hemodynamic instability, as is communication with the blood bank to facilitate adequate supply of blood products. Intraoperative autotransfusion has been shown to be effective and safe during liver transplantation, and its use may be considered when the $\mathrm{PRBC}$ requirement is $>5$ units. Its use is not recommended for patients with peritoneal infection or malignancy (Liang et al. 2008).

When lower cardiac output and/or hypotension persists even with adequate preload, dopamine or epinephrine may be infused for patients with hypotension, while dobutamine can be used when patients are normotensive. High venous pressures (CVP and PCWP) may be seen in patients with volume overload, large ascites, and pleural or pericardial effusion. Drainage of ascites and effusion may decrease intrathoracic pressure and central venous pressures and improve cardiac performance. Thoracentesis and pericardiocentesis can be performed after the abdomen is opened to minimize the risk of injury to the thoracoabdominal organs.

Unexpected pulmonary hypertension may be observed in some patients. Because of the high perioperative mortality in patients with pulmonary hypertension, it deserves a thorough intraoperative investigation. The PA catheter should be able to differentiate between pulmonary hypertension with high pulmonary vascular resistance and pulmonary hypertension with fluid overloading. Pulmonary hypertension caused by fluid overloading may dissipate gradually by intraoperative fluid loss, and phlebotomy may be 
required in severe hypervolemia. In portopulmonary hypertension with increased pulmonary vascular resistance, the presence of right ventricular function is investigated. A low cardiac output with a high CVP suggests the presence of right ventricular dysfunction. TEE findings of right ventricle dysfunction are low fractional area change (FAC), tricuspid annular plane excursion (TAPSE) of $<16 \mathrm{~mm}$, flattening of the ventricular septum, apicalization of the right ventricle, and right ventricular dilation. Additionally, the pulmonary vascular response to various vasodilators (i.e., diltiazem, nitroglycerin, epoprostenol, and nitric oxide) may be evaluated. Liver transplantation may continue when pulmonary hypertension is mild to moderate with normal right ventricular function. In such cases, right ventricular function is supported by maintaining optimal preload and improving myocardial contractility by inotropes.

Complications of massive blood transfusion (ionic hypocalcemia, ionic hypomagnesemia, hyperkalemia, and acidosis) may develop at this stage and should be treated aggressively. Normothermia can be well-maintained even during massive transfusion when a rapid-infusion device is used.

\section{Anhepatic Stage}

Hemodynamic changes that occur during the anhepatic stage are caused primarily by interruption of venous return from the IVC and portal vein. In the simple cross-clamping technique, clamping of the IVC and portal vein reduces venous return by up to $40 \%$, leading to low cardiac output, hypotension, and compensatory tachycardia (Pappas et al. 1971). Calculated systemic vascular resistance is frequently elevated, although this is a reflection of the exclusion of the vascular tree of the lower extremities and splanchnic bed. It is noteworthy that cross-clamping of the IVC and the portal vein decreases the central blood volume and pressure (CVP and PCWP) but progressively increases total intravascular blood volume as blood is sequestered in the vascular bed of the gastrointestinal and pelvic organs, kidneys, and lower extremities. A prolonged low output state, portal hypertension, and renal venous congestion may lead to acidosis, intestinal swelling, and hematuria.
We, at Thomas Jefferson University Hospital, prefer to treat the low output state by administration of fluid and/or inotropes (dopamine or dobutamine $2-5 \mu \mathrm{g} / \mathrm{kg} / \mathrm{min})$.

Venovenous bypass is more physiologic technique than a simple cross-clamping technique as it returns venous blood from the portal and IVC system (Shaw et al. 1984). Hence, hemodynamic changes that occur during the anhepatic stage with venovenous bypass are minimal when the bypass flow rate is greater than $25 \%$ of the baseline cardiac output and, therefore, the bypass flow should be monitored and adjusted as needed. Improper positioning of the cannula tip in the femoral or portal vein, or a kinked bypass circuit, may not drain blood adequately and the surgical team should correct their positions. A low pump speed reduces venous return, while a high pump speed collapses the outflow venous wall and decreases the bypass flow. The perfusionist, therefore, should adjust the pump speed to maximize the bypass flow. In addition, hypovolemia decreases the bypass flow, and it should be corrected by the anesthesia team. The anesthesia and surgical teams should be prepared for potential acute complications of venovenous bypass. Bleeding or air entry may result from venous laceration during cannulation or improperly secured cannulas. Entry of a small volume of air (up to $50 \mathrm{~mL}$ ) into the bypass pump may not cause immediate systemic air embolism because it is trapped in the cone-shaped pump head by centripetal force. Thromboembolism may be caused by the migration of pre-existing thrombi or those developed during a low bypass flow rate $(<1000 \mathrm{~mL} / \mathrm{min})$, particularly in hypercoagulable conditions (i.e., Budd-Chiari syndrome, neoplasms, and congenital protein $\mathrm{C}$ deficiency). Most importantly, the bypass may have to be terminated unexpectedly when serious complications occur. Therefore, the anesthesia team should be prepared for unexpected crossclamping of the IVC and portal vein at all times. After completion of the IVC anastomosis, the portal cannula is removed to facilitate the portal venous anastomosis, resulting in partial bypass. Low bypass flow and low cardiac output during this period can be improved by the 
administration of fluids or dopamine, but full correction of central hypovolemia, as reflected on CVP, PCWP, or TEE, is avoided to prevent fluid overload on reperfusion.

In the original description of the piggyback technique, adequate venous return is maintained through the intact IVC and portal vein using portoaxillary venovenous bypass (Tzakis et al. 1989). Currently, many transplantation centers do not incorporate the portoaxillary venovenous bypass in the piggyback technique, which makes patients vulnerable to significant hypovolemia. Hepatectomy in the presence of portal hypertension can be difficult, and hypovolemia is not uncommon as a consequence of inadvertent compression of the IVC and portal vein, partial side-clamping of the IVC, and cross-clamping of the portal vein during portal anastomosis. Hence, temporary portacaval shunt or portal-axillary venovenous bypass may be instituted in surgically challenging patients to maintain preload.

As described earlier, $1000 \mathrm{~mL}$ of cold lactated Ringer's solution or albumin (5\%) is flushed through the portal vein and drained via the incompletely anastomosed IVC to remove preservative solution, metabolites, and air from the allograft. Additionally, approximately $300-500 \mathrm{~mL}$ of blood may be allowed to escape through the incompletely anastomosed portal vein to enhance the washout by partial unclamping of the infrahepatic IVC (back-bleeding technique) immediately before reperfusion of the grafted liver. In this case, blood should be administered simultaneously to avoid hypovolemia.

Other factors that affect circulation during the anhepatic stage are similar to those of the dissection stage, although lactic acidosis, citrate intoxication, hypomagnesemia, hyperkalemia, and coagulopathy are more pronounced. Hyperkalemia is treated by dextrose $(5-10 \mathrm{~g})$ and insulin (5-10 units) to move potassium intracellularly (DeWolf et al. 1993a). In severe hyperkalemia, PRBC or phlebotomized blood can be washed using an autotransfusion system to remove potassium before transfusion (Ellis et al. 1987). At the end of the anhepatic stage, all biochemical variables are normalized to prepare for reperfusion.

\section{Neohepatic Stage}

Significant hemodynamic changes occur on reperfusion of the grafted liver (Fig. 6). Unclamping of the infrahepatic IVC and portal vein results in transient hypovolemia and hypotension due to acute sequestration of the blood in the engrafted liver. Unclamping of the suprahepatic IVC increases preload by mobilizing blood from the low extremities and splanchnic circulation. This is followed by severe hemodynamic changes, the so-called postreperfusion syndrome (Aggarwal et al. 1993). The postreperfusion syndrome, which occurs in approximately $30 \%$ of patients, is defined by abrupt hypotension (below $70 \%$ of the baseline value) that develops within 5 min of reperfusion and lasts for more than $1 \mathrm{~min}$. Other associated hemodynamic changes are bradycardia, high CVP and PCWP, low systemic vascular resistance, and conduction defects. Acute reduction in myocardial contractility is observed in TEE. The postreperfusion syndrome appears to be caused by a combination of several factors. For example, an acute increase in preload may result in right ventricular strain and an acute decrease in blood temperature $\left(2-3{ }^{\circ} \mathrm{C}\right)$ by the systemic entry of the cold preservation solution may decrease cardiac conduction and contractility. Other physical factors are air embolism and thromboembolism, which may cause right ventricular strain or right ventricular outflow tract obstruction (Ellis et al. 1989; Suriani et al. 1996). Chemical factors involved are acute hyperkalemia and acidosis. Systemic entry of hyperkalemic preservation solution increases serum potassium level to a very high level (up to $12 \mathrm{mmol} / \mathrm{L}$ ), causing severe bradycardia and conduction defects (Martin 1986). Return of the acidic blood from the viscera and lower extremities increases the base deficit by $5-10 \mathrm{mmol} / \mathrm{L}$. In addition, unknown endogenous vasodilators or myocardial depressants (i.e., vasoactive intestinal polypeptide, nitric oxide, and eicosanoid) released from the allograft or congested viscera may decrease systemic vascular resistance and impair myocardial function.

Several measures may be taken to prevent the postreperfusion syndrome, although they are not always successful. At the end of the anhepatic 


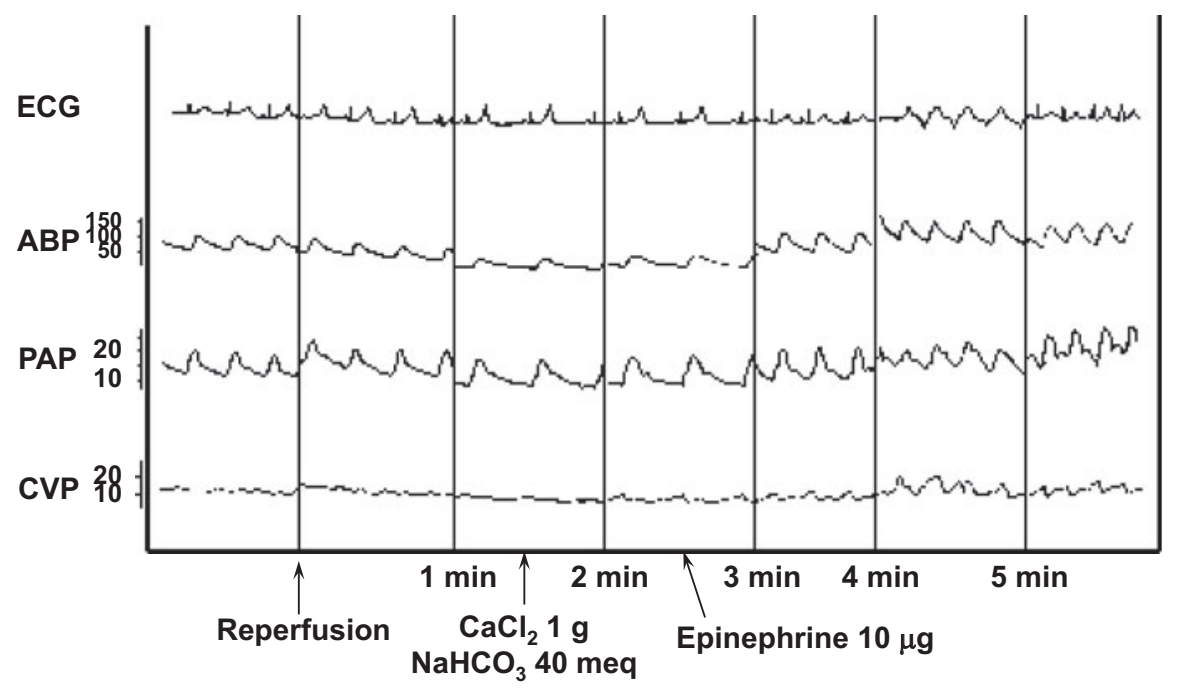

Fig. 6 Postreperfusion syndrome and its treatment (This article was published in Kang YG, Gelman S, Liver transplantation. In: Gelman S (ed) Anesthesia and organ transplantation. WB Saunders, Philadelphia, Copyright Elsevier (1987))

stage, blood volume is adjusted to avoid fluid overloading on reperfusion, and ionic hypocalcemia, hyperkalemia, and metabolic acidosis are corrected. Prophylactic administration of $\mathrm{CaCl}_{2}$ $(15 \mathrm{mg} / \mathrm{kg}), \mathrm{NaHCO}_{3}(0.5-1 \mathrm{mmol} / \mathrm{kg})$, regular insulin (10 units), $50 \%$ dextrose $(1 \mathrm{~mL} / \mathrm{kg})$, and epinephrine $(5-10 \mu \mathrm{g})$ are recommended by some centers (Ellis et al. 1989). Once the postreperfusion syndrome develops, severe hypotension and bradycardia are treated with small doses of epinephrine ( $5 \mu \mathrm{g}$ increments) to support contractility, heart rate, and vasomotor tone, followed by a dopamine or epinephrine infusion, if necessary. Symptomatic hyperkalemia (tall, peaked-T wave, and widening QRS complex with bradycardia) is treated by administration of $\mathrm{CaCl}_{2}(15 \mathrm{mg} / \mathrm{kg})$ and $\mathrm{NaHCO}_{3}(0.5-1 \mathrm{mmol} /$ $\mathrm{kg}$ ). Arrhythmias are treated in the standard fashion. When pulmonary edema develops, positive end-expiratory pressure (PEEP) is applied and inotropes may be given. Patients who develop intracardiac or pulmonary embolism are supported by inotropes. When severe fluid overloading is a concern, phlebotomy may be considered.

The postreperfusion syndrome dissipates gradually over the next 5-15 min, although low systemic vascular resistance and hypotension with a high cardiac output may persist for several hours. When hypotension is suspected to cause tissue and myocardial ischemia, it may be treated with ephedrine, dopamine, or epinephrine. Overzealous administration of fluids may result in hepatic congestion, while norepinephrine may interfere with hepatic blood flow by decreasing portal venous flow. Octreotide and vasopressin may increase arterial blood pressure by decreasing portal pressure and flow, although its effects on hepatic circulation and metabolism are unclear (Fayed et al. 2013; Wagener et al. 2008). Hemodynamic changes that occur during hepatic arterial and biliary reconstruction are relatively minor, except for intermittent fluctuation of the preload associated with continuous third-space fluid loss and compression of the liver and great vessels.

\section{Pulmonary Homeostasis}

Gas exchange is maintained satisfactorily in most patients. Minute volume is gradually decreased during the anhepatic stage to match the reduced oxygen consumption and carbon dioxide production, and is increased during the neohepatic stage. Alveolar recruitment maneuvers are performed intermittently to avoid atelectasis caused by 
pleural effusions, cephalad traction of the rib cage, and compression of diaphragm. Intermittent endotracheal suctioning, using a suction catheter or bronchoscope, may be required to remove secretions. Drainage of pleural effusions and ascites decreases intrathoracic pressure and improves oxygenation within $2 \mathrm{~h}$. Patients with preoperative ARDS may require a high $\mathrm{FiO}_{2}$ and a high level of PEEP to ensure adequate gas exchange, and a volume ventilator may be necessary to overcome the high airway pressure. Frank pulmonary edema can develop, particularly after reperfusion, from the increased pulmonary capillary permeability or fluid overload. In such cases, patients are ventilated with a high level of $\mathrm{FiO}_{2}$ and PEEP, while the underlying cause is treated. Closure of the abdominal cavity may interfere with ventilation by increasing intrathoracic and airway pressures. Primary closure with mesh or secondary closure may be necessary.

\section{Cerebral Homeostasis}

In patients with fulminant hepatic failure, preoperative cerebral monitoring is continued as cerebral hyperemia and intracranial hypertension persist during surgery, although they are somewhat attenuated by general anesthetics. However, a sudden increase in preload may dramatically exacerbate intracranial hypertension on reperfusion of the grafted liver. Hence, optimal preload should be maintained during the entire procedure. After reperfusion, cerebral hyperemia may gradually decrease as the liver begins to function.

\section{Coagulation}

Intraoperative changes in coagulation are summarized in Table 4. Surgical bleeding is common due to numerous collateral vessels associated with portal hypertension, difficulty in dissection of the diseased liver, pre-existing coagulopathy, and pathologic changes in coagulation. The average blood loss in adults is 5-15 units each of PRBC and FFP, although blood loss may reach more than 100 units each.
During the dissection stage, dilutional coagulopathy develops as bleeding reduces the levels of coagulation factors and platelets (Fig. 7). Fibrinolysis may develop, particularly in patients with hepatocellular disease, as a result of a low level of inhibitors of fibrinolysis and impaired hepatic clearance of tPA (Lewis et al. 1989a). Excessive activation of coagulation, evidenced by a gradual increase in thrombin-antithrombin complex, develops at the end of the dissection stage (Kratzer et al. 1991). Management of coagulation begins with normalization of physiologic variables, such as ionic hypocalcemia, hypothermia, and acidosis impair coagulation (Rohrer and Natale 1992). This is followed by continuous infusion of coagulation factor-rich blood (RBC:FFP: PlasmaLyte-A $^{\circledR}$ or normal saline $=1$ unit:1 unit: $250 \mathrm{~mL}$ ) to maintain coagulation factor levels above the critical level (30-50\% of normal). Specific blood components may be administered based on TEG/ROTEM. In general, platelets (5-10 units) are administered for a small maximum amplitude (MA) $(<40 \mathrm{~mm})$. Platelet administration, in addition to increasing MA, improves reaction time $(r)$ and clot formation rate $(\alpha)$, because the coagulation cascade leading to fibrin formation occurs on the surface of platelets. Its administration, however, is withheld during the anhepatic stage to avoid potential thrombosis and during massive blood transfusion ( $>150 \mathrm{~mL} / \mathrm{min})$ to minimize wastage. Two units of FFP may be administered when the reaction time is prolonged ( $>12 \mathrm{~min}$ ) even after platelet administration Cryoprecipitate (6 units) containing factors I and VIII are rarely required unless severe fibrinolysis is left untreated because plasmin selectively destroys factors I, V, and VIII. However, cryoprecipitate may be used for patients with severe hypofibrinogenemia $(<100 \mathrm{mg} / \mathrm{dL})$.

Pathologic coagulation superimposes on dilutional coagulopathy during the anhepatic stage. The heparin effect is seen as a prolonged aPTT and reaction time on TEG/ROTEM at the onset of the venovenous bypass as a small dose of heparin (2000-5000 units) in the bypass circuit enters systemic circulation. This heparin effect dissipates over the next 30-60 min. The effects of the absence of the hepatic synthetic and 
Table 4 Intraoperative changes in coagulation

\begin{tabular}{l|l|l|l|l}
\hline & \multirow{2}{*}{} & \multirow{2}{*}{} & & \multicolumn{2}{l}{ Neohepatic stage } \\
\cline { 3 - 6 } & Dissection stage & Anhepatic stage & Early & \multicolumn{2}{l}{ Late } \\
\hline Pre-existing coagulopathy & ++ & ++ & ++ & + \\
\hline Dilution & +++ & +++ & ++ & + \\
\hline Hypocalcemia & + & +++ & ++ & + \\
\hline Hypothermia & + & ++ & +++ & + \\
\hline Fibrinolysis & + & ++ & ++++ & - \\
\hline Excessive coagulation & + & ++ & ++++ & - \\
\hline Heparin effect & - & $+^{\mathrm{a}}$ & ++ & - \\
\hline
\end{tabular}

${ }^{a}$ In patients with venovenous bypass with heparin in the priming solution. + mild increase; ++ moderate increase; +++ severe increase; and - no change

clearance function begin to develop during this stage. The absence of hepatic clearance of tPA promotes fibrinolysis in approximately $30 \%$ of patients (Kang et al. 1987). Similarly, the absence of hepatic clearance of activated coagulation factors results in excessive activation of coagulation evidenced by a progressive increase in thrombin-antithrombin complex and fibrin (ogen) degradation products. Severe fibrinolysis (fibrinolysis time $<60 \mathrm{~min}$ ) may be treated by the administration of a single, small dose of EACA (250-500 mg) (Kang et al. 1987). Administration of a large or repeated dose of EACA is not recommended in order to avoid potential thromboembolism (Gologorsky et al. 2001).

The postreperfusion syndrome occurs in coagulation at the onset of the neohepatic stage. A typical coagulation profile shows prolonged PT, aPTT, reptilase time, and thrombin time. A generalized decrease in coagulation factors (I, V, VII, and VIII) and platelets is accompanied by a sharp increase in the tPA level, a shortened euglobulin lysis time, and a moderate increase in fibrin(ogen) degradation products and thrombin-antithrombin complex. Fibrinolysis is observed in up to $80 \%$ of patients and is severe in about $40 \%$ (Kang et al. 1987). Fibrinolysis is caused by a 20 -fold increase in tPA being released from the allograft and congested viscera, which overwhelms the activity of the plasminogen activator inhibitor (Virji et al. 1989; Porte et al. 1989). There are ample data to support the finding that fibrinolysis is primary in origin: a relatively steady antithrombin level, only moderate levels of fibrin(ogen) degradation products and D-dimers, selective

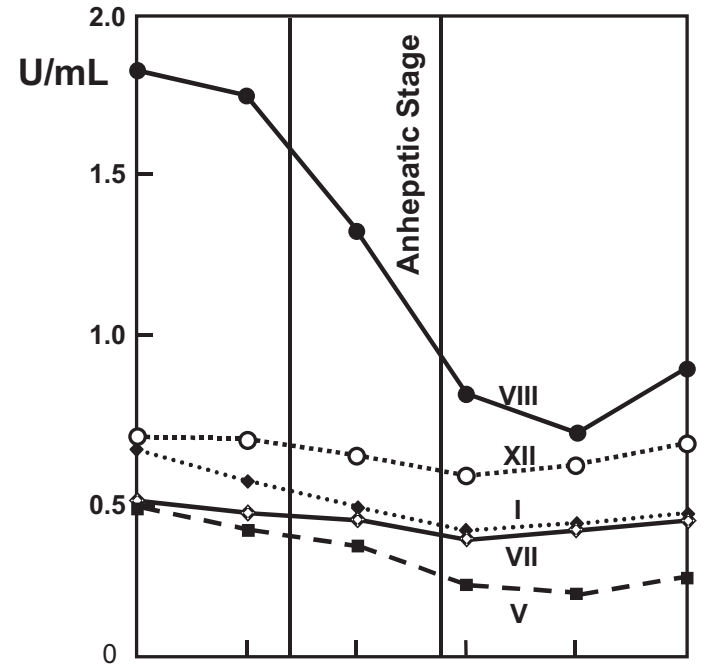

Time

Fig. 7 Intraoperative change in coagulation of 100 patients (Modified from Lewis et al. (1989a), with the permission of the publisher)

decreases in factors I, V, and VIII, and no known microthrombi formation (Lewis et al. 1989a, b). Fibrinolysis resolves over the 120 min following reperfusion. The heparin effect occurs in approximately $30 \%$ of patients, as heparin is released from the allograft and dissipates over the next 60-90 min.

To identify the presence of fibrinolysis and the heparin effect, TEG/ROTEMs of untreated blood (native), blood treated with antifibrinolytic agent (EACA or aprotinin), and blood treated with an agent neutralizing heparin (protamine sulfate or heparinase) are compared 5 min after reperfusion. When fibrinolysis is present, early treatment using 
a single, small dose of EACA (250-500 mg) is recommended in order to reduce delayed oozing and to minimize the loss of factors I, V, and VIII (Kang et al. 1997). Prophylactic administration of EACA or tranexamic acid (AMCA) is a common practice in many centers, but has not shown scientific efficacy. Fibrinolysis prophylaxis is not recommended by the authors, because the presence of fibrinolysis can easily be detected by TEG/ROTEM and can be treated effectively with a small dose of EACA in most patients. When the heparin effect is present, a small dose of protamine sulfate $(25-50 \mathrm{mg}$ ) may be given in severe cases. In addition, blood coagulability can be impaired by reperfusion hypothermia, acidosis, and ionic hypocalcemia.

In contrast, excessive activation of coagulation leading to fatal intracardiac or pulmonary embolism may occur in some patients (Gologorsky et al. 2001; Warnaar et al. 2008). This complication appears to be associated with a massive transfusion, release of a large quantity of tissue thromboplastin from the less than optimal allograft, impaired tissue perfusion, and possibly antifibrinolytic therapy. Intracardiac thrombosis can be treated by infusion of tPA (40-100 mg over $2 \mathrm{~h}$ ) while observing resolution of thrombi using TEE (Boone et al. 2011; Jackson et al. 2006).

Coagulopathy improves gradually after reperfusion. Generalized oozing, however, may occur even in the presence of acceptable coagulation profiles and TEG/ROTEM, possibly due to delayed bleeding caused by the loss of a poorly formed clot or by the residual effects of reperfusion fibrinolysis.

Several other pharmacologic agents are reported to improve coagulation. Aprotinin $(2,000,000 \mathrm{KIU}$ followed by $500,000 \mathrm{KIU} / \mathrm{h}$ ), a non-specific inhibitor of plasminogen and serine protease, may reduce blood loss by inhibiting fibrinolysis and excessive activation of coagulation (Neuhaus et al. 1989; Cottam et al. 1991). However, clinical use of aprotinin declined even before the drug was withdrawn by the manufacturer: clinical reports did not show a significant reduction in blood loss (Ickx et al. 1993; Groh et al. 1993), and fibrinolysis can be treated with EACA or AMCA more efficiently with negligible side effects (Kang et al. 1987; Boylan et al. 1996). Recombinant factor VIIa (rFVIIa) has been suggested to improve coagulation and reduces bleeding by actively enhancing coagulation and stimulating fibrin formation in the presence of tissue factor. Its beneficial effects have been shown in patients with fulminant hepatic failure, "critical bleeding," and a ruptured liver (Meadows et al. 2011; Merchant et al. 2004; Yamaguchi et al. 2015). However, results of clinical trials are controversial (Planinsic et al. 2005; Gasperi and Baudo 2006; Niemann et al. 2006) and a European consensus concluded that a paucity of data from clinical trials with rFVIIa limits both the strength and the scope of clinical recommendations (Vincent et al. 2006). Recently, the use of prothrombin complex concentrate has been assessed in a limited number of centers. Prothrombin complex concentrate is prepared from FFP and contains clotting factors II, VII, IX, and X, protein C, and protein $\mathrm{S}$, and its use may improve coagulation without increasing preload (Arshad et al. 2013). However, it has limited components of coagulation and its clinical advantage requires further investigation. Desmopressin acetate (DDAVP), a synthetic analog of 8-arginine vasopressin, increases the endothelial release of factor VIII, von Willebrand factor, and plasminogen. Its beneficial effects have been demonstrated in vitro and in patients with liver disease, and it may be used to improve coagulation $(0.3 \mu \mathrm{g} / \mathrm{kg}) \quad$ (Kang et al. 1993). Conjugated estrogen has been reported to improve coagulation and reduce blood loss (Frenette et al. 1998), although its use has not been accepted widely.

\section{Electrolyte and Acid-Base Homeostasis}

\section{Calcium Metabolism}

Patients with hepatic dysfunction invariably develop ionic hypocalcemia during massive blood transfusion, which is caused by chelation of serum calcium with citrate in the banked blood. Ionic hypocalcemia begins to appear during the dissection stage (Marquez et al. 1986) and becomes severe during the anhepatic stage. The serum-ionized calcium level is inversely related to 


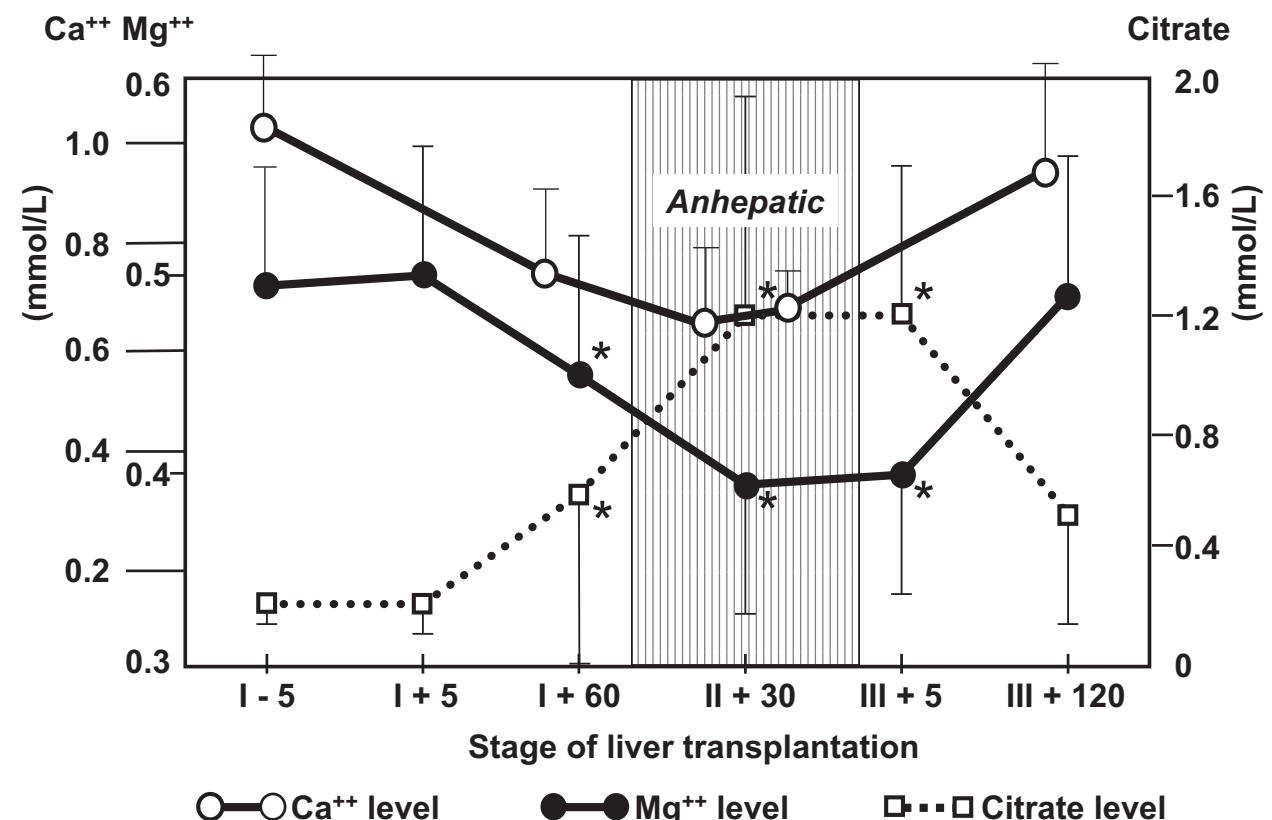

Fig. 8 Intraoperative changes in serum calcium, magnesium, and citrate level (Results of two studies (Marquez et al. 1986 and Scott et al. 1996) are superimposed, with the permission of the publisher)

the serum citrate level, as the absence of hepatic metabolism of citrate increases the serum citrate level close to that in the banked blood (Fig. 8). Significant hypocalcemia $\left(\mathrm{Ca}^{2+}<0.55 \mathrm{mmol} / \mathrm{L}\right)$ is associated with a prolonged $\mathrm{Q}-\mathrm{T}$ interval and decreases in the cardiac index, stroke-work index, and blood pressure. Therefore, the ionized calcium concentration is monitored hourly or more frequently, and $\mathrm{CaCl}_{2}(15 \mathrm{mg} / \mathrm{kg})$ or calcium gluconate $(30 \mathrm{mg} / \mathrm{kg})$ is administered to maintain a normal level (Martin et al. 1990). Ionic hypocalcemia improves gradually as the engrafted liver begins to metabolize citrate, unless the speed of the transfusion exceeds the metabolic function of the liver.

\section{Potassium Metabolism}

Hypokalemia is not uncommon in patients with liver disease due to poor dietary intake of potassium and its loss from chronic diuretic therapy and diarrhea. Severe hypokalemia $(<2.5 \mathrm{mmol} / \mathrm{L})$ is treated with potassium chloride to increase its level to $3.0-4.0 \mathrm{mmol} / \mathrm{L}$. Moderate hypokalemia $(<3.5 \mathrm{mmol} / \mathrm{L})$ is not treated because it is welltolerated by patients and self-corrected by blood transfusion. Hyperkalemia is a serious concern because it interferes with myocardial conduction and contractility, particularly in the presence of acidosis and hypocalcemia. Progressive hyperkalemia (up to $6-7 \mathrm{mmol} / \mathrm{L}$ ) may occur in patients with renal dysfunction or those requiring massive blood transfusion. Mild hyperkalemia (up to $5.5 \mathrm{mmol} / \mathrm{L}$ ) is treated with insulin (10 units) and glucose (12.5 g). It has been shown that glucose and insulin therapy is effective in lowering the serum potassium level even in the absence of hepatic function (Dewolf et al. 1993a). For moderate-to-severe hyperkalemia $(>5.5 \mathrm{mmol} / \mathrm{L})$, in addition to insulin therapy, PRBC or phlebotomized blood can be washed to remove potassium using an autotransfusion system before transfusion (Ellis et al. 1987).

Reperfusion hyperkalemia is caused by potassium influx from the preservation solution and hepatocytes, and its systemic effects and treatment have been described previously. Acute hyperkalemia returns to a normal range within 5-10 $\mathrm{min}$ as a result of redistribution. The potassium level gradually returns to the baseline value as the RBCs and the engrafted 
liver take up excess potassium. Hypokalemia $(<3.5 \mathrm{mmol} / \mathrm{L})$, which occurs toward the end of procedure, is treated using a $\mathrm{KCl}$ infusion (20 mmol increments).

\section{Sodium Metabolism}

Hyponatremia $(<130 \mathrm{mmol} / \mathrm{L})$ is a common occurrence in patients with liver disease, particularly those with fluid retention, ascites, diuretic therapy, and restricted sodium diet. The serum sodium level gradually increases towards normal during surgery via administration of blood products and a balanced salt solution. A rapid rise in the serum sodium level $(>10 \mathrm{mmol} / \mathrm{L})$ is a clinical concern because it may contribute to the development of central pontine myelinolysis, a serious neurological injury caused by the destruction of the myelin sheath in the pons (Videira et al. 1991). Therefore, the preoperative serum sodium level should be raised to $>130 \mathrm{mmol} / \mathrm{L}$, if possible, and a rapid increase in sodium should be prevented by administration of low sodium-containing crystalloids during surgery. In addition, tromethamine (THAM) is the preferred drug for treatment of metabolic acidosis as it does not contain sodium. Hypernatremia may be seen in some patients who receive a large dose of $\mathrm{NaHCO}_{3}$ preoperatively. This hypernatremia is gradually normalized by administration of blood products and a balanced electrolyte solution.

\section{Ionic Hypomagnesemia}

A clinical investigation showed that the serum ionized magnesium level, similar to the ionized calcium level, has an inverse relationship with the serum citrate level as magnesium ion chelates with citrate in banked blood (Scott et al. 1996). Although the clinical significance of ionic hypomagnesemia during liver transplantation is unclear, $\mathrm{MgSO}_{4}(1-4 \mathrm{~g})$ can be administered to minimize potential cardiac irritability and myocardial depression.

\section{Metabolic Acidosis}

Metabolic acidosis begins to appear during the dissection and anhepatic stages because of impaired hepatic metabolism of the acid load from the banked blood and the peripheral tissues. The base deficit and lactate level increase further (approximately $5 \mathrm{mmol} / \mathrm{L}$ ) on reperfusion due to the acid load from the graft and congested viscera and lower extremities. It gradually improves as hepatic function is restored and tissue perfusion improves during the neohepatic stage. Persistent lactic acidosis ( $>15 \mathrm{mmol} / \mathrm{L}$ ) appears to be associated with graft dysfunction (Begliomini et al. 1989).

Metabolic acidosis is aggressively corrected by administration of $\mathrm{NaHCO}_{3}$ to maintain base deficit levels $<5 \mathrm{mmol} / \mathrm{L}$ because acidosis is frequently progressive and leads to myocardial depression, inadequate cellular respiration, and decreased sensitivity to catecholamines. As described earlier, THAM is preferred in hypo- or hypernatremic conditions to minimize fluctuation of the serum sodium level: $150 \mathrm{~mL}$ of $0.3 \mathrm{M}$ THAM is equivalent to $50 \mathrm{mmol}$ of $\mathrm{NaHCO}_{3}$. Alternatively, dichloroacetate $(40 \mathrm{mg} / \mathrm{kg}$ every $4 \mathrm{~h}$ ) appears to reduce lactate production by stimulating pyruvate oxidation (Shangraw and Robinson 1997).

\section{Metabolic Alkalosis}

Metabolic alkalosis may develop during the neohepatic stage, and this was believed to be associated with $\mathrm{NaHCO}_{3}$-administered and citrate metabolism-generating bicarbonate. However, it has been shown that the degree of metabolic alkalosis is unrelated to the citrate and $\mathrm{NaHCO}_{3}$ load (Fortunato et al. 1987) and may be associated with residual hyperaldosteronism.

\section{Metabolic Homeostasis}

Body temperature may gradually decrease to $34{ }^{\circ} \mathrm{C}$ during the dissection stage as a result of the exposure of the abdominal contents to the cold environment, vasodilatation, and lack of shivering. Hypothermia continues during the anhepatic stage as energy production decreases further. An abrupt decrease in core temperature $\left(2-3{ }^{\circ} \mathrm{C}\right)$ occurs on reperfusion as cold preservation solution enters systemic circulation. The temperature increases during the neohepatic stage, and the surgery ends with a body temperature of 
approximately $35-36{ }^{\circ} \mathrm{C}$. Hypothermia is difficult to avoid, although raising the room temperature, application of forced warm air devices, use of a warming blanket, and a heat exchanger in the venovenous bypass system may be beneficial.

The blood glucose level is relatively wellmaintained (100-200 mg/dL) with blood transfusion, as the banked blood contains glucose (approximately $200 \mathrm{mg} / \mathrm{dL}$ ). A gradual decrease in glycogenolysis reduces the blood glucose level during the dissection and anhepatic stages. In patients with fulminant hepatic failure or severe hepatocellular disease, the blood glucose level may decrease precipitously, making glucose supplementation necessary. Hyperglycemia (up to $300 \mathrm{mg} / \mathrm{dL}$ ) occurs on reperfusion as glucose is released from the engrafted liver (DeWolf et al. 1987). Insulin does not appear to be effective in treating reperfusion hyperglycemia because glucose reuptake requires restoration of hepatic function. The insulin level is relatively steady during surgery, and the glucagon level increases after reperfusion. The blood glucose level usually returns to normal within 12-24 h. Persistent hyperglycemia caused by impaired hepatic glucose reuptake and hormonal imbalance is an early sign of poor graft function (Mallett et al. 1989).

\section{Renal Homeostasis}

Urine output is well-preserved in most patients once the intravascular volume is optimized. Oliguria or anuria, however, may persist in patients with the hepatorenal syndrome or underlying renal disease. The presence of oliguria and hematuria during the anhepatic stage of the simple cross-clamping technique has been described earlier. Urine output increases during the neohepatic stage as a result of the restoration of renal function and circulation. Various agents have been tried to protect or improve renal function: the role of dopamine is controversial, dopexamine appears to be beneficial, and triple-drug therapy (dopamine [2-3 $\mu \mathrm{g} / \mathrm{kg} / \mathrm{min}$ ], mannitol [250 $\mathrm{mg} / \mathrm{kg}$ ], and furosemide) improves urine output but not renal function (Gray et al. 1991; Planinsic et al. 1997). When fluid overload or severe electrolyte imbalance is a concern, intraoperative venovenous ultrafiltration or hemodialysis may be utilized.

\section{Conclusion of Surgery}

The restoration of hepatic function is evident about $2 \mathrm{~h}$ after reperfusion: levels of citrate and lactate decrease, the glucose level returns toward normal, coagulopathy improves, and bile production begins. Persistent citrate intoxication, acidosis, hyperglycemia, coagulopathy, and palecolored bile are poor prognostic signs.

Recently, tracheal extubation in the operating room has been successful in several centers when the patient meets the liver transplantation-specific extubation criteria, including the severity of pre-existing liver disease, blood loss, and hemodynamic stability (Mandell et al. 2002; Biancofiore et al. 2005; Glanemann et al. 2007). However, most patients are still transported to the intensive care unit (ICU) while receiving invasive monitoring and ventilatory support. Upon arrival to the ICU, the ventilator setting is reported to the respiratory therapist, the lungs are auscultated, and vital signs are displayed on the ICU monitor. Detailed intraoperative information is reported to the ICU physician and nursing staff.

\section{Postoperative Complications}

\section{Hepatic Complications}

\section{Primary Non-Function}

Primary non-function is defined as graft failure occurring within 90 days after liver transplantation in the absence of either rejection or technical factors such as hepatic arterial thrombosis (Bzeizi et al. 1997). This complication occurs in up to $10 \%$ of patients and is frequently caused by hepatic dysfunction of the donor liver or prolonged cold ischemia $(>18 \mathrm{~h})$. The patient develops progressive multi-organ failure including encephalopathy, coagulopathy, minimal bile production, and oliguria. Supportive therapy may 
be helpful until the liver resumes its function, although urgent retransplantation is the only solution in many patients.

\section{Acute Rejection}

Worsening liver function without technical complications in the second week after liver transplantation suggests acute cellular rejection. Biopsy findings are inflammation of the intrahepatic endothelium and bile duct and a mononuclear cell infiltration with eosinophilia (Wiesner 1996).

\section{Vascular Complications}

Hepatic arterial stenosis occurs in approximately $5 \%$ of patients, and is four times more common in children. Common clinical signs are biliary tract breakdown, recurrent bacteremia, hepatic abscess, and occasionally massive hepatic necrosis (Tzakis et al. 1985). Hepatic arterial stenosis is suspected when an ultrasound examination reveals increased focal arterial flow velocities and is confirmed by angiography. In the immediate postoperative period, direct repair or reconstruction using an infrarenal arterial conduit is usually successful. Stenosis occurring several weeks after transplantation is treated with percutaneous hepatic arterial angioplasty, which has a success rate of more than $90 \%$ in achieving long-term patency.

Vena caval stenosis and thrombosis occur in $1-2 \%$ of patients. In traditional liver transplantation, outflow obstruction is managed by balloon angioplasty with or without a metallic stent placement (Simo et al. 1993). In the piggyback technique, it is treated with end-to-side anastomoses between the donor infrahepatic IVC and the recipient retrohepatic IVC (Stieber et al. 1997).

Portal venous stenosis and thrombosis are relatively uncommon in the adult population and present with graft dysfunction, massive ascites formation, and hemodynamic instability. This complication is corrected by an urgent reconstruction of the portal vein or construction of a superior mesenteric venous graft to the liver, together with a ligation of large collaterals that may reduce the portal flow.

\section{Biliary Complications}

Biliary complications are more common in children and have an overall incidence of $8-15 \%$. Early recognition is difficult, leading to high morbidity and mortality. Bile leaks usually occur at the anastomotic site, although they may be found at the T-tube site or aberrant ducts. Most biliary complications occur within the first 3 months and are diagnosed by liver function tests (serum bilirubin, $\gamma$-glutamyltransferase, and alkaline phosphatase) and imaging techniques. These complications are treated by percutaneous or endoscopic drainage of bile collections. In cases of Roux-en-Y choledochojejunostomy, surgical reconstruction is required.

\section{Intra-Abdominal Bleeding}

Intra-abdominal bleeding occurs in about 7-15\% of patients and requires exploration in about half of these cases (Ozaki et al. 1994). Gastrointestinal bleeding may develop from ulcers, viral enteritis, varices, and an afferent Roux-en-Y loop. Variceal bleeding is usually associated with portal vein thrombosis and requires an urgent ultrasound or angiographic evaluation. Bleeding from the Roux-en-Y limb occurs 1 week after surgery and is usually self-limited. Additionally, bleeding can be caused by persistent thrombocytopenia associated with splenic sequestration, drug toxicity, heparin-induced thrombocytopenia, and immunologic reactions.

\section{Intestinal Perforation}

Intestinal perforation is caused by serosal injury to the intestines and usually occurs in patients who have had a technically difficult hepatectomy, prolonged portal venous clamping, or a massive blood transfusion. Intestinal perforation or leakage is treated by urgent surgery and antifungal therapy.

\section{Extrahepatic Complications}

\section{Cardiac Complications}

Any type of cardiac complication can develop in the postoperative period. Hypotension can occur due to hypovolemia, either from under- 
resuscitation or ongoing bleeding, decrease in contractility secondary to the pre-existing myocardial disease, or new onset of dilated or ischemic cardiomyopathy. Other potential causes are acidosis, hypocalcemia, or vasodilation from sepsis or graft failure. Management of cardiac complications is based on the underlying cause.

Hypertension occurs in patients with pre-existing hypertension, inadequate pain control, hypoglycemia, and cerebral edema. Restoration of normal liver function may increase systemic vascular resistance, and calcineurin inhibition can increase systemic blood pressure. Calcium channel blockers (i.e., diltiazem and verapamil) are avoided because they can increase the levels of the calcineurin inhibitors.

Myocardial infarction is relatively rare due to thorough preoperative evaluation being undertaken to detect CAD. However, when it does develop, a cardiologist should be consulted for possible emergent cardiac catheterization during surgery and revascularization. Pulmonary edema is commonly seen postoperatively and may be caused by significant transfusion requirements, increased capillary permeability, prolonged intubation, and reversible dilated cardiomyopathy.

Reversible dilated cardiomyopathy with pulmonary edema may develop in the first 5 days after transplantation. Sampathkumar et al. reported that $1 \%$ of patients who did not have ventricular dysfunction developed dilated cardiomyopathy postoperatively, most of whom recovered completely without any long-term complications (Sampathkumar et al. 1998). The cause of this condition is unknown, although it may be a form of stress-induced cardiomyopathy.

Atrial fibrillation, ventricular tachycardia, and other arrhythmias may develop as a result of electrolyte abnormalities (i.e., hypomagnesemia, hyperkalemia, and hypocalcemia), cardiac ischemia, or irritation from CVP or PA catheters. In a study by Xia et al., atrial fibrillation was observed in $7.4 \%$ of patients and was associated with increased mortality, graft failure, and acute kidney injury (Xia et al. 2015). All arrhythmias are treated following the standard guidelines.

Thromboembolism is a common cause of sudden postoperative death. Deep vein thrombosis should be prevented by early extubation and mobilization, use of compressive stockings, and administration of heparin (subcutaneous or low molecular weight).

\section{Pulmonary Complications}

Most patients require mechanical ventilation for only a few hours or days after transplantation. However, prolonged ventilatory support is required in some patients with atelectasis, pleural effusions, and central nervous system (CNS) depression. Intraoperative cross-clamping of the IVC occasionally results in right phrenic nerve crush injury and diaphragmatic paralysis in the immediate postoperative period (McAlister et al. 1993). ARDS may develop in patients with intra-abdominal infection, pancreatitis, hepatic necrosis, acute cellular rejection, and occasionally with Muromonab-CD3 (OKT3) treatment. Bronchoalveolar lavage and bacterial culture are frequently performed to rule out pulmonary infection from any other pulmonary pathology. Pre-existing pulmonary hypertension may persist postoperatively and is controlled by epoprostenol or nitroglycerin.

\section{Neurological Complications}

Neurological complications occur in 12-20\% of patients, mostly in the first week of transplantation (Singh et al. 1994). These are more common in adults and present as mental status changes ranging from dysphasia to frank coma. Dysfunction of the CNS is commonly caused by medications, such as cyclosporine, tacrolimus, histamine $\mathrm{H}_{2}$-blockers, acyclovir, and antibiotics such as imipenem. Non-convulsive seizures may occur, and an EEG is performed for patients with unexplained mentation changes. Intracranial hemorrhage and watershed infarcts are ruled out using CT scans. Hyponatremia and hypomagnesemia can also delay awakening. Central pontine myelinolysis may develop several days after transplantation, and recovery is often slow and incomplete (Winnock et al. 1993). Hepatic encephalopathy may be present for several days after transplantation in patients with persistent portosystemic shunting. Meningitis should be ruled out when the mental status change is accompanied by fever. Disseminated aspergillosis is a 
devastating complication in a patient with multiple brain infarcts and fever. Peripheral neuropathy presenting as weakness is usually myopathic in nature and is more common in patients with preoperative severe liver disease, poor graft function, high steroid doses, and uremia, and are confirmed by electromyography and muscle biopsy.

In patients with fulminant hepatic failure, cerebral hyperemia and hypertension usually decrease gradually, and the patient regains consciousness as the liver begins to function.

\section{Renal Dysfunction}

Renal dysfunction is usually transient and is commonly associated with intraoperative hypovolemia and hypotension, allograft dysfunction, and nephrotoxicity of cyclosporine and tacrolimus. Oliguria is an early sign of renal dysfunction and is managed by restoring intravascular volume and renal perfusion. The hepatorenal syndrome may persist after transplantation, and its recovery depends on its preoperative severity and allograft function. In some patients, addition of vasoconstrictive immunosuppressants (cyclosporine and tacrolimus) may lead to acute tubular necrosis. In general, renal function returns to the normal range in most patients, and approximately $10 \%$ of patients require temporary dialysis (McCaulley et al. 1990). Long-term prognosis is fair, although hypertension, diabetes, and chronic nephropathy induced by steroids and the calcineurin inhibitors may result in chronic renal failure.

\section{Infectious Complications}

More than half of the postoperative infections following liver transplantation are bacterial in origin. These infections typically occur in the first 2 weeks, when blood levels of immunosuppressants are high. The most common sites of infection are the liver, biliary tract, peritoneal cavity, and pulmonary system. Common organisms in the abdomen are aerobic Gram-positive organisms (Streptococci and Staphylococci) and Gramnegative bacilli (Escherichia coli, Enterobacter species, and Pseudomonas), while Pseudomonas infection is most common in the lungs. Approximately $20 \%$ of infections are caused by fungus, with Candida species accounting for more than
$80 \%$ of all fungal infections. The risk factors are a high steroid dosage, usage of broad-spectrum antibiotics, and prolonged surgical time. Candida infection is treated with amphotericin or fluconazole. Aspergillus infection accounts for $15 \%$ of all fungal infections and is associated with a very high mortality; high-dose liposomal amphotericin B followed by prolonged itraconazole is the treatment of choice. Viral infections are seen 2-3 months after transplantation, with cytomegalovirus and herpes simplex accounting for the bulk of these infections. Epstein-Barr virus is not usually seen until approximately 6 months after transplantation, but is an important cause of lymphoproliferative disease. Pneumocystis pneumonia, an opportunistic infection, responds to trimethoprim-sulfamethoxazole.

\section{Late Metabolic Complications}

Late metabolic complications following liver transplantation include diabetes, hyperlipidemia, weight gain, and hypertension. Diabetes is induced by steroids, cyclosporine, and tacrolimus and may respond to oral hypoglycemic agents or insulin. Hyperlipidemia is associated with diabetes, obesity, steroids, and immunosuppressive drugs and is treated by diet and exercise. Hypertension is seen in as many as $85 \%$ of patients after transplantation; the use of steroids or tacrolimus is the most likely cause. Hypomagnesemia has been implicated as the cause of the hypertension in some cases.

\section{Anesthesia for Specific Conditions}

\section{Retransplantation of the Liver}

Approximately $15 \%$ of all patients require retransplantation of the liver. Early retransplantation is performed within several days after the primary transplantation to rescue patients from primary non-function (graft factor), acute rejection, and technical failure (vascular thrombosis), or secondary non-function (host factor) associated with poor hepatic perfusion. Hepatic necrosis is the common pathway of graft non-function and results in progressive, severe encephalopathy, ARDS, lactic acidosis, 
coagulopathy, hypoglycemia, and significant circulatory instability. Although infrequent, hepatectomy with a portacaval shunt may be performed to protect the patient from the ill effects of the necrotizing liver on extrahepatic organ functions. In such a case, retransplantation should be performed as soon as the donor organ is available. The surgical procedure itself is relatively simple because surgical dissection has already been made and adhesions have not yet formed. Anesthetic management of these patients is similar to that of patients undergoing primary transplantation.

Late retransplantation is performed in patients with chronic rejection, vascular complications, and recurrence of the original disease. The physical condition of the patient may have improved, but complications of immunosuppression (i.e., hypertension, renal insufficiency) may be present. Adhesions and the steroid-induced fragile tissues frequently complicate late retransplantation. Anesthetic management is similar to that of primary liver transplantation, but a large amount of blood loss is anticipated.

\section{Pediatric Liver Transplantation}

In pediatric liver transplantation, rapid-sequence IV induction is preferred, although mask induction is chosen in patients in whom there is difficulty obtaining IV access (Borland et al. 1985). Large-bore IV catheters are placed in the upper extremities after induction of anesthesia. A central venous catheter with CVP monitoring is the usual procedure, and pulmonary arterial catheterization is rarely indicated. Blood pressure is monitored using a femoral intra-arterial catheter. It appears that children tolerate cross-clamping of the IVC and portal vein reasonably well without significant hemodynamic changes, possibly by compliant vasomotor tone. Therefore, venovenous bypass is rarely used in children under $20 \mathrm{~kg}$. Coagulation changes that occur during liver transplantation are not as severe as those of adults, and this may be associated with more prevalent cholestatic diseases in children (Kang et al. 1989). Blood loss in children with biliary atresia can be large due to the technical difficulty associated with previous biliary surgery (i.e., Ksai procedure). Maintenance of body temperature is difficult, as the large surface area promotes heat loss.

\section{Live-Donor Hepatectomy}

Live-donor hepatectomy is usually a challenging procedure. The young and healthy donors (ASA Physical Status [PS] 1 or 2) undergo a complete evaluation by hepatologists, surgeons, anesthesiologists, and psychologists. The anesthetic goals are minimizing surgical blood loss and allogeneic blood transfusion, maintaining liver blood flow, facilitating early extubation, preventing deep venous thrombosis and infection, and providing adequate postoperative pain control. Preoperatively, donors may be given erythropoietin to boost $\mathrm{RBC}$ production and they can donate 2 units of autologous whole blood 2-3 weeks before surgery. On the day of surgery, donors may be given heparin (5000 units, subcutaneous injection) to prevent deep venous thrombosis, and 6 units of typed and cross-matched PRBCs are prepared. In the holding area, a peripheral IV catheter is secured, anxiolytics are administered, and donors may elect to receive thoracic epidural anesthesia for postoperative analgesia. The need for epidural local anesthetics with or without narcotics is determined by the attending anesthesiologist and pain service.

In the operating room, Unasyn ${ }^{\circledR}$ (3 g IV) or vancomycin (if allergic to penicillin) is administered to prevent infection, and the patient is positioned with minimal stress to the brachial plexus to avoid neurologic injury (Dulitz et al. 2005). Induction and maintenance of anesthesia follows the standard guidelines of any major surgical procedure. Ultra-short-acting narcotics such as remifentanil may be beneficial for early extubation after surgery as it is rapidly metabolized by plasma esterase and does not have a prolonged effect in the presence of hepatic and renal dysfunction.

Intraoperative monitoring is similar to that of patients undergoing major surgery, and a radial arterial catheter and CVP are placed for hemodynamic monitoring. Additional IV access is secured 
to prepare for the potential need for rapid infusion of fluids using a rapid-infusion system. Immediately after induction of anesthesia, isovolemic hemodilution may be performed: 2 units of the patient's whole blood is collected in CPDA (citrate phosphate dextrose adenine) blood collection bags, agitated to prevent clot formation, stored at room temperature, and returned to the patient within $8 \mathrm{~h}$.

Intraoperatively, physiologic condition should be maintained at all times to ensure adequate perfusion of all tissues including the liver by monitoring the cardiopulmonary system and stat laboratory. Metabolic acidosis should be avoided, and use of a balanced salt solution (lactated Ringer's solution or PlasmaLyte- $A^{\circledR}$ ) is the preferred choice in order to avoid the acid load from normal saline (Waters et al. 2001). Blood loss is not excessive and pre- and intraoperatively donated autologous blood and intraoperative autotransfusion are sufficient in most patients. The relationship between the CVP level and surgical blood loss is controversial: Chhibber et al. reported that intraoperative blood loss did not correlate with CVP $(<5 \mathrm{mmHg})$ in their study (Chhibber et al. 2007), while Jones et al. demonstrated a significant reduction in blood loss with low CVP (Jones et al. 1998). The authors recommend euvolemia to maintain hepatic blood flow during dissection of the liver. However, fluid overloading should be avoided after hepatectomy because relatively high portal venous flow to the reduced liver mass may lead to liver congestion and small-for-size syndrome (Dahm et al. 2005). At the conclusion of surgery, the patient can be extubated safely in the operating room and transported to the ICU.

\section{Surgery After Liver Transplantation}

All types of surgical procedures may be necessary in the early postoperative period. Within the first 2 months after transplantation, surgical procedures are performed to treat complications of transplantation, such as exploratory laparotomy for abdominal bleeding or reconstruction of the biliary system. Some degree of hepatic dysfunction may still be present, and ventilatory and circulatory support and invasive monitoring may be required. Regional anesthesia is not recommended because of potential bleeding and infectious complications. Anesthesia care of these patients is similar to that of other urgent abdominal procedures.

Patients may return to the operating room at any time for biliary reconstruction, replacement of a hip joint, or almost any other procedure. Liver function and drug metabolism are usually within the normal range, and anesthetic management differs little from that of other patients. Side effects of immunosuppressants (hypertension and renal insufficiency) and drug interactions should be considered.

\section{Cadaveric Donor Procurement}

The main goal of organ procurement is the maintenance of optimal conditions for all organ systems to promote as normal as possible an environment for the organs prior to harvesting. Specifically, integrity of organs should be maintained by optimizing organ perfusion and preventing further damage associated with pre-existing illness or trauma. Therefore, donor care during procurement is a continuum of the intensive care provided before brain death. The donor is reviewed and examined by the anesthesia team to evaluate their medical history and vital organ function.

The equipment and medications necessary for multiple organ procurement are shown in Table 5. A multiple-channel vital-sign monitor is an essential piece of equipment because of the unavoidable hemodynamic changes associated with the absence of brain stem function, surgical manipulation, and fluid shift. A volume ventilator may be required for donors requiring high levels of PEEP or airway pressure. A large volume of crystalloids and colloid solutions is prepared, and 5 units of PRBCs are frequently required. The transit from the ICU to the operating room is a crucial period; the anesthesia care team directs the transportation while the donor is continuously monitored, ventilated, and treated. 
Table 5 Anesthesia checklist for donor procurement

1. Family consent and permission from the coroner

2. Donor support guidelines

Systolic blood pressure $>100 \mathrm{mmHg}$

Central venous pressure $<12 \mathrm{cmH}_{2} \mathrm{O}$

Urine output $>100 \mathrm{~mL} / \mathrm{h}$ blood glucose

\begin{tabular}{l}
\hline 3. Equipment \\
\hline Transport monitor \\
\hline Anesthetic gas machine \\
\hline Warming blanket and blood warmer \\
\hline Defibrillator \\
\hline Multiple-channel vital-sign monitor \\
\hline Ventilator \\
\hline Infusion devices \\
\hline 4. Laboratory tests \\
\hline Hemoglobin and hematocrit \\
\hline Serum electrolytes, calcium, lactate \\
\hline Arterial blood gas tension and acid-base state \\
\hline 5. Medications \\
\hline Packed red blood cells $(5 \mathrm{units})$ \\
\hline Dopamine (400 mg) \\
\hline Methylprednisolone (30 mg/kg) \\
\hline Mannitol (25 \%, $100 \mathrm{~g})$ \\
\hline Lactated Ringer's solution (12-15 L) \\
\hline Heparin (20,000 units) \\
\hline Chlorpromazine (250 mg) \\
\hline Furosemide (100 mg) \\
\hline
\end{tabular}

Intraoperatively, blood pressure is monitored by an indwelling radial or brachial arterial catheter as abrupt changes in blood pressure are anticipated. CVP monitoring is essential, and a PA catheter may be used in unstable donors. General anesthesia is provided as donors respond to surgical stimulation by dramatic hemodynamic changes such as tachycardia, hypertension, perspiration, and involuntary movement (Wetzel et al. 1985). This so-called mass reflex is caused by the neurogenic vasoconstriction and stimulation of adrenal medulla by reflex spinal arc. Isoflurane is the most commonly used agent, because its myocardial depression is relatively benign, and short-acting narcotics (i.e., fentanyl, up to $5 \mu \mathrm{g} / \mathrm{kg} / \mathrm{min}$ ) may be used in unstable donors. Rocuronium bromide or vecuronium bromide is administered for muscle relaxation.

The specific goals of ventilatory care are to maintain normal $\mathrm{PaO}_{2}(70-100 \mathrm{mmHg})$, arterial hemoglobin oxygen saturation (>95\%), and $\mathrm{PaCO}_{2}(35-45 \mathrm{mmHg})$ as well as to avoid pulmonary complications. This goal is frequently achieved by ventilating with a tidal volume of $10-15 \mathrm{~mL} / \mathrm{kg}, \mathrm{FiO}_{2}$ of $30-40 \%$, respiratory rate of $<20 / \mathrm{min}$, and a low level of PEEP $\left(<5 \mathrm{cmH}_{2} \mathrm{O}\right)$. However, in donors with pulmonary complications, adjustments are made in tidal volume (up to $20 \mathrm{~mL} / \mathrm{kg}$ ), respiratory rate (up to 20/min), and PEEP (up to $10 \mathrm{cmH}_{2} \mathrm{O}$ ).

Aggressive circulatory care is essential because hemodynamic instability may impair organ perfusion. Specifically, hypotension (systolic blood pressure $<80 \mathrm{mmHg}$ or mean arterial pressure $<40 \mathrm{mmHg}$ ) is associated with a high incidence of acute tubular necrosis, non-function of the graft kidneys, and poor hepatic function. It is generally agreed that systolic blood pressure should be within the normal range $(100-120 \mathrm{mmHg})$ and CVP should be $<10 \mathrm{cmH}_{2} \mathrm{O}$ with minimal vasopressor support. Maintaining circulatory homeostasis, however, can be challenging. Preload is frequently decreased because of blood loss, vasomotor paralysis, diuretic therapy, and diabetes inspidus, although fluid resuscitation may result in overload. The heart rate may vary depending on the degree of brain injury, ranging from tachycardia to bradycardia. Arrhythmia is not uncommon, and myocardial contractility is frequently impaired by myocytolysis, myocardial necrosis, coronary spasm, and reduction of myocardial energy storage (Novitzky et al. 1988). Afterload may be high, from excessive sympathetic tone, or low, from vasomotor paralysis. Volume deficit is usually corrected with lactated Ringer's or colloid solution, and transfusion of PRBCs (1-3 units) may be necessary to maintain hematocrit between 25 and $35 \%$ (Hardesty and Griffith 1986). Once the fluid deficit is corrected, a glucose-containing hypotonic solution ( $5 \%$ dextrose in $0.45 \% \mathrm{NaCl}$ $1 \mathrm{~mL} / \mathrm{kg} / \mathrm{h}$ ) is administered to replace urine output and insensible loss, guided by CVP and urine output. Excessive urine output ( $>200-250 \mathrm{~mL} / \mathrm{h}$ ) is replaced using a hypotonic electrolyte solution with supplementation of $\mathrm{KCl}(20 \mathrm{mEq} / \mathrm{L})$. Tachycardia with hypertension should be avoided as it may cause pulmonary edema, decrease organ perfusion, and increase myocardial oxygen 
consumption. A $\beta$-antagonist (i.e., labetalol hydrochloride or esmolol hydrochloride) or a calcium channel blocker (verapamil hydrochloride) is used to treat tachycardia and arrhythmia (Novitzky et al. 1984). For bradycardia, isoproterenol or epinephrine is used for positive chronotropic effects because donors are unresponsive to centrally acting chronotropic drugs (i.e., atropine). Supraventricular or ventricular arrythmia is treated using antiarrhythmic drugs. Low afterload is compensated for by increasing preload because $\alpha$-vasopressors increase the myocardial work load and decrease splanchnic and coronary blood flow. In severely hypertensive donors, an $\alpha$-blocker (hydralazine or sodium nitroprusside) may be given to reduce the afterload. When cardiac output and organ perfusion are impaired, inotropes (dopamine hydrochloride, dobutamine hydrochloride, and isoproterenol hydrochloride) are recommended to improve cardiac contractility. In brain-dead animal models, serum levels of triiodothyronine, insulin, and cortisol have been found to be low, and the administration of triiodothyronine may improve hemodynamic stability by maintaining myocardial high-energy stores and glycogen (James et al. 2010). Circulatory arrest, which occurs in $10 \%$ of potential donors (Emery et al. 1986), is managed in the standard fashion, except atropine is not effective.

Adequate diuresis $(>0.5 \mathrm{~mL} / \mathrm{kg} / \mathrm{h}$, preferably $1-1.5 \mathrm{~mL} / \mathrm{kg} / \mathrm{h}$ ) is recommended as urine output $(>100 \mathrm{~mL} / \mathrm{h})$ is the most significant factor that determines the outcome of the kidney and liver graft. Oliguria is generally caused by hypovolemia and hypotension and frequently responds to fluid administration. Diabetes insipidus leads to polyuria, hypovolemia, and electrolyte imbalance. In addition to the fluid replacement, DDAVP (0.5-1 units/h) may be administered (Richardson and Robinson 1985), although an excessive dose of DDAVP may increase the risk of acute tubular necrosis and reduce hepatic blood flow (Burggraaf et al. 1994).

Donors are poikilothermic, and hypothermia plays a major role in hemodynamic instability. Body temperature should be kept above $35^{\circ} \mathrm{C}$ by raising the operating room temperature, infusing all fluids through a blood warmer, and using heating lamps, a warming blanket, and a heated humidifier in the ventilation circuit. Metabolic acidosis, caused by inadequate tissue perfusion, is corrected by administration of $\mathrm{NaHCO}_{3}$ or THAM. Commonly seen electrolyte imbalances are hypernatremia, hypokalemia, hypocalcemia, hypophosphatemia, and hypomagnesemia, and they are treated in a standard fashion. Glucose metabolism is relatively well-maintained, and any abnormality in glucose metabolism is corrected by administration of insulin or glucose on the basis of the serum glucose level.

Dilutional coagulopathy is common, and consumption coagulopathy may develop secondary to the release of tissue thromboplastin from injured tissues and the ischemic organs (Kaufman et al. 1984). Fibrinolysis is not uncommon in donors, possibly as a result of the release of tPA from the necrotic brain. Replacement of coagulation factors and platelets or any pharmacologic therapy is rarely indicated as donors are fully heparinized when the aorta is cannulated. Once cardiac arrest is induced by cardioplegia, no further supportive care is necessary.

\section{Conclusion}

Liver transplantation is one of the most stressful procedures for patients with multiple organ dysfunction and it is a challenge for anesthesiologists. It is remarkable that anesthesiologists have played a major role in the progress of liver transplantation and its successful outcome. It cannot be overemphasized, however, that a thorough understanding of pathophysiology and close communication and cooperation among hepatologists, surgeons, anesthesiologists, intensivists, and other healthcare workers are vital to successful outcomes and further progress in this field.

\section{Cross-References}

Hepatopulmonary Syndrome and
Portopulmonary




\section{References}

Aggarwal S, Kang Y, Freeman JA et al (1993) Postreperfusion syndrome: hypotension after reperfusion of the transplanted liver. J Crit Care 8:154-160

Aggarwal S, Kramer D, Yonas H et al (1994) Cerebral hemodynamic and metabolic changes in fulminant hepatic failure: a retrospective study. Hepatology 19:80-87

American Society of Anesthesiologists. Guidelines for director of liver transplant anesthesia. http://www. asahq.org/ /media/Sites/ASAHQ/Files/Public/Resources/ standards-guidelines/guidelines-for-director-of-liver-trans plant-anesthesia.pdf. Accessed 16 Jun 2016

American Society of Anesthesiologists (2015) Practice guidelines for perioperative blood management: an updated report by the American Society of Anesthesiologists Task Force on Perioperative Blood Management. Anesthesiology 122:241-275

Arroyo V, Gines A, Gerbes A et al (1996) Definition and diagnostic criteria of refractory ascites and hepatorenal syndrome in cirrhosis. Hepatology 23:164-176

Arshad F, Ickx B, van Beem RT et al (2013) Prothrombin complex concentrate in the reduction of blood loss during orthotopic liver transplantation: PROTONtrial. BMC Surg 13:22

Begliomini B, DeWolf A, Freeman J et al (1989) Intraoperative lactate levels can predict graft function after liver transplantation [abstract]. Anesthesiology 71:A72

Benoit JN, Barrowman JA, Harper SL et al (1984) Role of humoral factors in the intestinal hyperemia associated with chronic portal hypertension. Am J Physiol 247: G486-G493

Biancofiore G, Bindi ML, Romanelli AM et al (2005) Fast track in liver transplantation: 5 years' experience. Eur J Anaesthesiol 22:584-590

Bismuth H (1982) Surgical anatomy and anatomical surgery of the liver. World J Surg 6:3-9

Boone JD, Sherwani SS, Herborn JC et al (2011) The successful use of low-dose recombinant tissue plasminogen activator for treatment of intracardiac/pulmonary thrombosis during liver transplantation. Anesth Analg 112:319-321

Borland LM, Roule M, Cook DR (1985) Anesthesia for pediatric orthotopic liver transplantation. Anesth Analg 64:117-124

Boylan JF, Klinck JR, Sandler AN et al (1996) Tranexamic acid reduces blood loss, transfusion requirements, and coagulation factor use in primary orthotopic liver transplantation. Anesthesiology 85:1043-1048

Burger-Klepp U, Karatosic R, Thum M et al (2012) Transesophageal echocardiography during orthotopic liver transplantation in patients with esophagoastric varices. Transplantation 94:192-196

Burggraaf J, Schoemaker HC, Kroon JM et al (1994) Influence of 1-desamino-8-D-vasopressin on endogenous fibrinolysis, haemodynamics and liver blood flow in healthy subjects. Clin Sci (Lond) 86(5):497-503
Butterworth RF (2001) Neurotransmitter dysfunction in hepatic encephalopathy: new approaches and new findings. Metab Brain Dis 16:55-65

Bzeizi KI, Jalan R, Plevris JN et al (1997) Primary graft dysfunction after liver transplantation: from pathogenesis to prevention. Liver Transpl Surg 3:137-148

Calne R (ed) (1983) Liver transplantation: the Cambridge/ King's College Hospital experience. Grune \& Stratton, London

Caramelo C, Fernandes-Munoz D, Santos JC et al (1986) Effect of volume expansion on hemodynamics, capillary permeability and renal function in conscious cirrhotic rats. Hepatology 6:129-134

Carey WD, Dumor JA, Pimentel RR et al (1995) The prevalence of coronary artery disease in liver transplant candidates over age 50. Transplantation 59:859-864

Cassagneau PI, Jacquier A, Giorgi R et al (2012) Prognostic value of preoperative coronary computed tomography angiography in patients treated by orthotopic liver transplantation. Eur J Gastroenterol Hepatol 24:558-562

Chait A, Brunzell JD (1990) Acquired hyperlipidemia (secondary dyslipoproteinemias). Endocrinol Metab Clin North Am 19:259-278

Charlton MR, Wall WJ, Ojo AO et al (2009) Report of the first international liver transplantation society expert panel consensus conference on renal insufficiency in liver transplantation. Liver Transpl 15:S1-S34

Chhibber A, Dziak J, Kolano J et al (2007) Anesthesia care for adult live donor hepatectomy: our experiences with 100 cases. Liver Transpl 13:537-542

Cottam S, Hunt B, Segal H, Ginsburg R, Potter D (1991) Aprotinin inhibits tissue plasminogen activatormediated fibrinolysis during orthotopic liver transplantation. Transplant Proc 23(3):1933

Couinaud C (1954) Lobes et segments hepatiques: notes sur I'architecture anatomique et chirurgicale de foie. Presse Med 62:709-712

D’Souza MG, Plevak DJ, Kvols L et al (1993) Elevated neuropeptide levels decrease during liver transplant. Transplant Proc 25:1805-1806

Dahm F, Georgiev P, Clavien PA (2005) Small-for-size syndrome after partial liver transplantation: definition, mechanisms of disease and clinical implications. Am J Transplant 5:2605-2610

DeWolf A, Kang YG, Todo S et al (1987) Glucose metabolism during liver transplantation in dogs. Anesth Analg 66:76-80

DeWolf A, Frenette L, Kang $\mathrm{Y}$ et al (1993a) Insulin decreases the serum potassium concentration during the anhepatic stage of liver transplantation. Anesthesiology 78:677-682

DeWolf AM, Begliomini B, Gasior RA et al (1993b) Right ventricular function during orthotopic liver transplantation. Anesth Analg 76:562-568

Doyle HR, Marino IR, Miro A et al (1993) Adult respiratory distress syndrome secondary to endstage liver 
disease-successful outcome following liver transplantation. Transplantation 55:292-296

Dzik WH, Jenkins R (1985) Use of intraoperative blood salvage during orthotopic liver transplantation. Arch Surg 120:946-948

Dulitz MG, DeWolf AM, Wong H et al (2005) Compression of the brachial plexus injury during right lobe liver donation as a cause of brachial plexus injury: a case report. Liver Transpl 11:233-235

Elia E, Kang Y (2002) Rapid infusion devices for hemorrhagic cardiothoracic trauma. Semin Cardiothorac Vasc Anesth 6:105-112

Ellis R, Beeston JT, Witherington SS et al (1987) Liver transplantation: effect of washing bank blood on intraoperative control of hyperkalemia. Transplant Proc 19(Suppl 3):73-74

Ellis JE, Lichtor JL, Feinstein SB et al (1989) Right heart dysfunction, pulmonary embolism, and paradoxical embolization during liver transplantation. A transesophageal two-dimensional echocardiographic study. Anesth Analg 68:777-782

Ellis RE, Yuan JY, Horvitz HR (1991) Mechanisms and functions of cell death. Annu Rev Cell Biol 7:663-698

Emery RW, Cork RC, Levinson MM et al (1986) The cardiac donor: a six year experience. Ann Thorac Surg 41:356-362

Fayed NI, Refaat EK, Yassein TE et al (2013) Effect of perioperative terlipressin infusion on systemic, hepatic, and renal hemodynamics during living donor liver transplantation. J Crit Care 28:775-782

Filsoufi F, Salzberg SP, Rahmanian PB et al (2007) Early and late outcome of cardiac surgery in patients with liver cirrhosis. Liver Transpl 13:990-995

Fluckiger M (1884) Vorkommen von trommelschlagelformigen fingerendphalangen ohne chronische Veranderungeng an den lungen oder am Herzen. Wien Med Wochenschr 34:1457-1462

Fortunato FL Jr, Kang Y, Aggarwal S et al (1987) Acid-base state during and after orthotopic liver transplantation. Transplant Proc 19(Suppl 3):59-60

Frenette L, Cox J, McArdle P et al (1998) Conjugated estrogen reduces transfusion and coagulation factor requirements in orthotopic liver transplantation. Anesth Analg 86:1183-1186

Gandhi CR, Kang Y, DeWolf A et al (1996) Altered endothelin homeostasis in patients undergoing liver transplantation. Liver Transpl Surg 2:362-369

Gasperi AD, Baudo F (2006) Use of recombinant factor VIIa during orthotopic liver transplantation. Liver Transpl 12:1176-1177

Gelman S, Ernst E (1977) Role of pH, $\mathrm{pCO}_{2}$, and $\mathrm{O}_{2}$ content of portal blood in hepatic circulatory autoregulation. Am J Physiol 233:E255-E262

Genovesi MG, Tierney DF, Taplin GA (1976) An intravenous radionuclide method to evaluate hypoxemia caused by abnormal alveolar vessels. Am Rev Respir Dis 114:59-65
Gerbes A, Remien J, Jungst D et al (1986) Evidence for down regulation of beta-adrenoceptors in cirrhotic patients with severe ascites. Lancet 1:1409-1410

Ghobrial RM, Amersi F, Busuttil RW (2000) Surgical advances in liver transplantation. Living related and split donors. Clin Liver Dis 4:553-565

Glanemann M, Hoffmeister R, Neumann U et al (2007) Fast tracking in liver transplantation: which patient benefits from this approach? Transplant Proc 39:535-536

Gologorsky E, De Wolf AM, Scott V et al (2001) Intracardiac thrombus formation and pulmonary thromboembolism immediately after graft reperfusion in 7 patients undergoing liver transplantation. Liver Transpl 7:783-789

Gray PA, Bodenham AR, Park GR (1991) A comparison of dopexamine and dopamine to prevent renal impairment in patients undergoing orthotopic liver transplantation. Anaesthesia 46:638-641

Groh J, Welte M, Azad SC et al (1993) Does aprotinin really reduce blood loss in orthotopic liver transplantation? Semin Thromb Haemost 19:306-308

Guevara M, Gines P, Fernandez-Esparrach G et al (1998) Reversal of hepatorenal syndrome by prolonged administration of ornipressin and plasma volume expansion. Hepatology 27:35-41

Gulati M, Cooper-DeHoff RM, McClure C et al (2009) Adverse cardiovascular outcomes in women with nonobstructive coronary artery disease: a report from the Women's Ischemia Syndrome Evaluation Study and the St James Women Take Heart Project. Arch Intern Med 169:843-850

Hardesty RL, Griffith BP (1986) Multiple cadaveric organ procurement for transplantation with emphasis on the heart. Surg Clin North Am 66:451-457

Hare JM, Colucci WS (1995) Role of nitric oxide in the regulation of myocardial function. Prog Cardiovasc Dis 38:155-166

Harinstein ME, Flaherty JD, Ansari AH et al (2008) Predictive value of dobutamine stress echocardiography for coronary artery disease detection in liver transplant candidates. Am J Transplant 8:1523-1528

Hourani LM, Bellamy PE, Tashkin DP et al (1991) Pulmonary dysfunction in advanced liver disease: frequent occurrence of an abnormal diffusing capacity. Am J Med 90:693-700

Ickx B, Pradier O, DeGroote F et al (1993) Effect of two different dosages of aprotonin on perioperative blood loss during liver transplantation. Semin Thromb Hemost 19:300-301

Jackson D, Boeta A, Gubenko Y et al (2006) Successful intraoperative use of recombinant tissue plasminogen activator during liver transplantation complicated by massive intracardiac/pulmonary thrombosis. Anesth Analg 102:724-728

James SR, Ranasinghe AM, Venkateswaran R et al (2010) The effects of acute triiodothyronine therapy on myocardial gene expression in brain stem 
dead cardiac donors. J Clin Endocrinol Metab 95:1338-1343

Jaue DN, Ma Z, Lee SS (1997) Cardiac muscarinic receptor function in rats with cirrhotic cardiomyopathy. Hepatology 25:1361-1365

Jones RM, Moulton CE, Hardy KJ (1998) Central venous pressure and its effect on blood loss during liver resection. Br J Surg 85:1058-1060

Kalayoglu M, Sollinger HW, Stratta RJ et al (1988) Extended preservation of the liver for clinical transplantation. Lancet 1:617-619

Kalb TH, Walter M, Mayer M et al (1993) Intra-allograft production and systemic release of tumor necrosis factor-alpha: detection upon reperfusion. Transplant Proc 25:1817-1818

Kang YG (1986) Monitoring and treatment of coagulation. In: Winter PM, Kang YG (eds) Hepatic transplantation, anesthetic and perioperative management. Prager, New York

Kang Y (1995) Thrombelastography in liver transplantation. Semin Thromb Haemost 21(Suppl 4):34-44

Kang Y (1997) Transfusion based on clinical coagulation monitoring does reduce hemorrhage during liver transplantation. Liver Transpl Surg 3:655-659

Kang YG, Gelman S (1987) Liver transplantation. In: Gelman S (ed) Anesthesia and organ transplantation. WB Saunders, Philadelphia

Kang YG, Lewis JH, Navalgund A et al (1987) Epsilonaminocaproic acid for treatment of fibrinolysis during liver transplantation. Anesthesiology 66:766-773

Kang Y, Borland LM, Picone J et al (1989) Intraoperative coagulation changes in children undergoing liver transplantation. Anesthesiology 71:44-47

Kang Y, Aggarwal S, Virji M et al (1991) Clinical evaluation of autotransfusion during liver transplantation. Anesth Analg 72:94-100

Kang Y, Scott V, DeWolf A et al (1993) In vitro effects of DDAVP during liver transplantation. Transplant Proc 25:1821-1822

Kang Y, Carranza JA, Chung CJ (1997) A low dose EACA during liver transplantation [abstract]. Liver Transpl Surg 3:C-46

Kaufman HH, Hui KS, Mattson JC et al (1984) Clinicopathologic correlations of disseminated intravascular coagulation in patients with severe head injury. Neurosurgery 15:34-42

Kawasaki T, Takeshita A, Souda K et al (1999) Serum thrombopoietin levels in patients with chronic hepatitis and liver cirrhosis. Am J Gastroenterol 94:1918-1922

Kemmer N, Case J, Chandna S, Neff GW (2014) The role of coronary calcium score in the risk assessment of liver transplant candidates. Transplant Proc 46:230-233

Kiuchi T, Kasahara M, Uryuhara K et al (1999) Impact of graft size mismatching on graft prognosis in liver transplantation from living donors. Transplantation 67 (2):321-327
Kjaer M, Jurlander J, Keiding S et al (1994) No reinnervation of hepatic sympathetic nerves after liver transplantation in human subjects. J Hepatol 20:97-100

Kojima H, Sakurai M, Kuriyama S et al (2002) Endothelin1 plays a major role in portal hypertension of biliary cirrhotic rats through endothelin receptor subtype B together with subtype A in vivo. J Hepatol 34:805-811

Kowalski HJ, Abelmann WH (1953) The cardiac output at rest in Laennec's cirrhosis. J Clin Invest 32:1025-1033

Kratzer MAA, Dieterich J, Denecke H et al (1991) Hemostatic variables and blood loss during orthotopic human liver transplantation. Transplant Proc 23:1906-1911

Krowka MJ, Cortese DA (1985) Pulmonary aspects of chronic liver disease and liver transplantation. Mayo Clin Proc 60:407-418

Krowka MJ, Wiseman GA, Burnett OL et al (2000) Hepatopulmonary syndrome: a prospective study of relationships between severity of liver disease, $\mathrm{PaO}$ (2) response to $100 \%$ oxygen, and brain uptake after (99m)Tc MAA lung scanning. Chest 118:615-624

Lamers WH, Hilberts A, Furt E et al (1989) Hepatic enzymic zonation: a reevaluation of the concept of the liver acinus. Hepatology 10:72-75

Lautt WW (1983) Relationship between hepatic blood flow and overall metabolism: the hepatic arterial buffer response. Fed Proc 42:1662-1666

Lautt WW (2007) Regulatory processes interacting to maintain hepatic blood flow constancy: vascular compliance, hepatic arterial buffer response, hepatorenal reflex, liver regeneration, escape from vasoconstriction. Hepatol Res 37:891-903

Lautt WW, Legare DJ, d'Almeida MS (1985) Adenosine as a putative regulator of hepatic arterial flow (the buffer response). Am J Physiol 248:H331-H338

Lebrec D, Capron J-P (1979) Pulmonary hypertension complicating portal hypertension. Am Rev Respir Dis 120:849-856

Lee SS (1989) Cardiac abnormalities in liver cirrhosis. West J Med 151:530-535

Lee SS, Marty J, Mantz J et al (1990) Desensitization of myocardial beta adrenergic receptors in cirrhotic rats. Hepatology 12:481-485

Lee M, Weinberg L, Pearce B et al (2015) Agreement between radial and femoral arterial blood pressure measurements during orthotopic liver transplantation. Crit Care Resusc 17:101-107

Lentine KL et al (2012) Cardiac disease evaluation and management among transplantation candidates: a scientific statement from the American Heart Association and the American College of Cardiology Foundation. Circulation 126:617-663

Lewis JH, Bontempo FA, Sa A et al (1989a) Liver transplantation: intraoperative changes in coagulation factors in 100 first transplants. Hepatology 9:710-714

Lewis JH, Bontempo FA, Ragni MV et al (1989b) Antithrombin III during liver transplantation. Transplant Proc 21:3543-3544 
Liang TB, Li DL, Liang L et al (2008) Intraoperative blood salvage during liver transplantation in patients with hepatocellular carcinoma: efficiency of leukocyte depletion filters in the removal of tumor cells. Transplantation 85:863-869

Lidorsky SD, BassNM PMC et al (1992) Intracranial pressure monitoring in liver transplantation for fulminant hepatic failure. Hepatology 16:1-7

Lieber CS (1993) Biochemical factors in alcoholic liver disease. Semin Liver Dis 13:136-153

Liu H, Lee SS (1999) Cardiopulmonary dysfunction in cirrhosis. J Gastro Hepatol 14:600-608

Liu H, Song D, Lee SS (2002) Cirrhotic cardiomyopathy. Gastroenterol Clin Biol 26:842-847

Ma Z, Miyamoto A, Lee SS (1996) Role of altered beta adrenoceptor signal transduction in the pathogenesis of cirrhotic cardiomyopathy in rats. Gastroenterology 110:1191-1198

Mallett SV, Kang Y, Freeman JA et al (1989) Prognostic significance of reperfusion hyperglycemia during liver transplantation. Anesth Analg 68:182-185

Mandell MS, Lezotte D, Kam I et al (2002) Reduced use of intensive care after liver transplantation: patient attributes that determine early transfer to surgical wards. Liver Transpl 8:682-687

Mantz FA, Craige E (1951) Portal axis thrombosis with spontaneous portocaval shunt and resulting cor pulmonale. Arch Pathol 52:91-97

Marquez J, Martin D, Kang YG et al (1986) Cardiovascular depression secondary to citrate intoxication during hepatic transplantation in man. Anesthesiology 65:457-461

Martin D (1986) Fluid and electrolyte balance during liver transplantation. In: Winter PM, Kang YG (eds) Hepatic transplantation, anesthetic and perioperative management. Prager, Philadelphia

Martin TJ, Kang Y, Robertson KM et al (1990) Ionization and hemodynamic effects of calcium chloride and calcium gluconate in the absence of hepatic function. Anesthesiology 73:62-65

Massicotte L, Perrault MA, Denault AY et al (2010) Effects of phlebotomy and phenylephrine infusion on portal venous pressure and systemic hemodynamics during liver transplantation. Transplantation 89:920-927

Matuschak GM, Shaw BW (1987) Adult respiratory distress syndrome associated with acute liver allograft rejection: resolution following hepatic transplantation. Crit Care Med 15:878-881

McAlister VC, Grant DR, Roy A et al (1993) Right phrenic nerve injury in orthotopic liver transplantation. Transplantation 55:826-830

McCaulley J, Van Thiel D, Starzl TE et al (1990) Acute and chronic renal failure after liver transplantation. Nephron 55:121-128

McDonnell DJ, Toye PA, Hutchins GM (1983) Primary pulmonary hypertension and cirrhosis: are they related? Am Rev Respir Dis 127:437-441
Meadows HB1, Krisl JC, Greenberg CS, et al (2011) Clinical usefulness of recombinant factor VII in patients with liver failure undergoing invasive procedures. Ann Pharmacother 45:1433-1438

Mehrabi A, Golling M, Kashfi A et al (2005) Negative impact of systemic catecholamine administration on hepatic blood perfusion after porcine liver transplantation. Liver Transpl 11:174-187

Melendez JA, Arslan V, Fischer ME et al (1998) Perioperative outcomes of major hepatic resections under low central venous pressure anesthesia: blood loss, blood transfusion, and the risk of postoperative renal dysfunction. J Am Coll Surg 187:620-625

Merchant SH, Mathew P, Vanderjagt TJ, et al (2004) Recombinant factor VIIa in management of spontaneous subcapsular liver hematoma associated with pregnancy. Obstet Gynecol 103:1055-8

Michalopoulos GK (1990) Liver regeneration: molecular mechanisms of growth control. FASEB J 4:176-187

Milani A, Zaccaria R, Bombardieri G et al (2007) Cirrhotic cardiomyopathy. Dig Liver Dis 39:507-515

Mulkay JP, Louis H, Donckier V et al (2001) Long term terlipressin administration improves renal function in patients with cirrhosis with type 1 hepatorenal syndrome: a pilot study. Acta Gastroenterol Belg 64:15-19

Neuhaus P, Bechstein WO, Lefebre B et al (1989) Effect of aprotinin on intraoperative bleeding and fibrinolysis in liver transplantation. Lancet 2:924-925

Niemann CU, Behrends M, Quan D et al (2006) Recombinant factor VIIa reduces transfusion requirements in liver transplant patients with high MELD scores. Transfus Med 16:93-100

Novitzky D, Wicomb MN, Cooper DKC et al (1984) Electrocardiographic, hemodynamic and endocrine changes occurring during experimental brain death in the chacma baboon. Heart Transplant 4:63

Novitzky D, Rose AG, Cooper DKC (1988) Injury of myocardial conduction tissue and coronary artery smooth muscle following brain death in the baboon. Transplantation 45:964-966

O'Rourke RA, Brundage BH, Froelicher VF et al (2000) American College of Cardiology/American Heart Association expert consensus document on electronbeam computed tomography for the diagnosis and prognosis of coronary artery disease. J Am Coll Cardiol 36:326-340

Ozaki CF, Katz SM, Monsour HP et al (1994) Surgical complications of liver transplantation. Surg Clin North Am 74:1155-1167

Pappas G, Palmer WM, Martineau GL et al (1971) Hemodynamic changes in clinical orthotopic liver transplantation. Surg Forum 22:335-336

Payen DM, Fratacci MD, Dupuy P et al (1990) Portal and hepatic arterial blood flow measurements of human transplanted liver by implanted Doppler probes: interest for early complications and nutrition. Surgery 107:417-427 
Petrides A, DeFronzo RA (1989) Glucose metabolism in cirrhosis: a review with some perspectives for the future. Diabetes Metab Rev 5:691-709

Pierrangelo A, Panduro A, Chowdhury JR et al (1992) Albumin gene expression is down regulated by albumin or macromolecule infusion in the rat. J Clin Invest 89:1755-1760

Pilkis SJ, Granner DK (1992) Molecular physiology of the regulation of hepatic gluconeogenesis and glycolysis. Annu Rev Physiol 54:885-909

Planinsic RM, Kang Y, DeWolf AM et al (1997) Dopamine, furosemide and mannitol infusions and changes in serum creatinines after liver transplantation. Liver Transpl Surg 3:10

Planinsic RM, van der Meer J, Testa G et al (2005) Safety and efficacy of a single bolus administration of recombinant factor VIIa in liver transplantation due to chronic liver disease. Liver Transpl 11:895-900

Plotkin JS, Benite RM, Kuo PC et al (1998) Dobutamine stress echocardiography for preoperative cardiac risk stratification in patients undergoing orthotopic liver transplantation. Liver Transpl Surg 4:253-257

Popper H (1977) Pathologic aspects of cirrhosis. Am J Pathol 87:228-264

Porte RJ, Bontempo FA, Knott EAR et al (1989) Systemic effects of tissue plasminogen activator-associated fibrinolysis and its relation to thrombin generation in orthotopic liver transplantation. Transplantation 47:978-984

Raval Z, Harinstein ME, Skaro AI et al (2011) Cardiovascular risk assessment of the liver transplant candidate. $\mathrm{J}$ Am Coll Cardiol 58:223-231

Richardson DW, Robinson AG (1985) Desmopressin. Ann Intern Med 103:228-239

Rohrer MJ, Natale AM (1992) Effect of hypothermia on the coagulation cascade. Crit Care Med 20:1402-1405

Rubin MH, Weston MJ, Langley MH et al (1979) Platelet function in chronic liver disease; relationship to disease severity. Dig Dis Sci 24:197-202

Rubin DA, Schulman DS, Edwards TD et al (1994) Myocardial ischemia after orthotopic liver transplantation. Am J Cardiol 74:53-56

Safadi A, Homsi M, Maskoun W et al (2009) Perioperative risk predictors of cardiac outcomes in patients undergoing liver transplantation surgery. Circulation 120:1189-1194

Sakai T, Planinsic MR, Hilmi IA et al (2007) Complications associated with percutaneous placement of venous return cannula for venovenous bypass in adult orthotopic liver transplantation. Liver Transpl 13:961-965

Sampathkumar P, Lerman A, Kim BY et al (1998) Postliver transplantation myocardial dysfunction. Liver Transpl Surg 4:399-403

Schroeder RA, Collins BH, Tuttle-Newhall E et al (2004) Intraoperative fluid management during orthotopic liver transplantation. J Cardiothorac Vasc Anesth $18: 438-441$
Scott VL, De Wolf AM, Kang Y et al (1996) Ionized hypomagnesemia in patients undergoing orthotopic liver transplantation: a complication of citrate intoxication. Liver Transpl Surg 2:343-347

Shah A, Variyam E (1988) Pericardial effusion and left ventricular dysfunction associated with ascites secondary to hepatic cirrhosis. Arch Intern Med 148:585-588

Shangraw RE, Robinson ST (1997) Oxygen metabolism during liver transplantation: the effect of dichloroacetate. Anesth Analg 85:746-752

Sharma M, Yong C, Majure D et al (2009) Safety of cardiac catheterization in patients with end-stage liver disease awaiting liver transplantation. Am J Cardiol 103:742-746

Shaw BW, Martin DJ, Marquez JM et al (1984) Venous bypass in clinical liver transplantation. Ann Surg 4:524-534

Shaw LJ, Raggi P, Schisterman E et al (2003) Prognostic value of cardiac risk factors and coronary artery calcium screening for all-cause mortality. Radiology 228:826-833

Siegerstetter V, Deibert P, Ochs A et al (2001) Treatment of refractory hepatic hydrothorax with transjugular intrahepatic portosystemic shunt: long term results in 40 patients. Eur J Gastroenterol Hepatol 13:529-534

Simo G, Echenagusia A, Camunez F et al (1993) (1993) Stenosis of the inferior vena cava after liver transplantation: treatment with Gianturco expandable metallic stents. Cardiovasc Intervent Radiol 18:212-216

Singh N, Yu VL, Gayowski T (1994) Central nervous system lesions in adult liver transplant recipients: clinical review with implications for management. Medicine 73:110-118

Smyrniotis V, Kostopanagiotou G, Kondi A et al (2002) Hemodynamic interaction between portal vein and hepatic artery flow in small-for-size split liver transplantation. Transpl Int 15(7):355-360

Snyder N, Atterbury CE, Correia JP et al (1977) Increased concurrence of cirrhosis and bacterial endocarditis. Gastroenterology 73:1107-1113

Sorkine P, Ben Abraham R, Szold O et al (2001) Role of the molecular adsorbent recycling system (MARS) in the treatment of patients with acute exacerbation of chronic liver failure. Crit Care Med 29:1332-1336

Starzl TE, Putnam CW (eds) (1969) Experience in hepatic transplantation. Saunders, Philadelphia

Starzl TE, Marchioro TL, von Kaulla KN et al (1963) Homotransplantation of the liver in humans. Surg Gynecol Obstet 117:659-676

Starzl TE, Todo S, Fung J et al (1989) FK 506 for liver, kidney, and pancreas transplantation. Lancet 28:1000-1004

Stieber AS, Gordon RD, Bassi N (1997) A simple solution to a technical complication in piggy back liver transplantation. Transplantation 64:654-655

Suriani RJ, Cutrone A, Feierman D et al (1996) Intraoperative transesophageal echocardiography 
during liver transplantation. J Cardiothorac Vasc Anesth 10:699-707

Tarter RE, Hegedus AM, Van Thiel DH et al (1984) Non alcoholic cirrhosis associated with neuropsychological dysfunction in the absence of overt evidence of hepatic encephalopathy. Gastroenterology 86:1421-1427

Tiukinhoy-Laing SD, Rossi JS, Bayram M et al (2006) Cardiac hemodynamic and coronary angiographic characteristics of patients being evaluated for liver transplantation. Am J Cardiol 98:178-181

Tzakis AG, Gordon RD, Shaw BW et al (1985) Clinical presentation of hepatic artery thrombosis after liver transplantation in the cyclosporine era. Transplantation 40:667-671

Tzakis A, Todo S, Starzl TE (1989) Orthotopic liver transplantation with preservation of the inferior vena cava. Ann Surg 210:649-652

Van Thiel DH, George M, Fareed J (2001) Low levels of thrombin activatable fibrinolysis inhibitor (TAFI) in patients with chronic liver disease. Thromb Haemost 85:667-670

Vaquero J, Fontana RJ, Larson AM et al (2005) Complications and use of intracranial pressure monitoring in patients with acute liver failure and severe encephalopathy. Liver Transpl 11:1581-1589

Videira R, Kang YG, Martinez J et al (1991) A rapid increase in sodium is associated with CPM after liver transplantation [abstract]. Anesthesiology 75: A222

Vincent JL, Rossaint R, Riou B et al (2006) Recommendations on the use of recombinant activated factor VII as an adjunctive treatment for massive bleeding-a European perspective. Crit Care 10:R120

Virji MA, Aggarwal S, Kang Y (1989) Alterations in plasminogen activator and plasminogen activator inhibitor levels during liver transplantation. Transplant Proc 21:3540-3541

Wagener G, Gubitosa G, Renz J et al (2008) Vasopressin decreases portal vein pressure and flow in the native liver during liver transplantation. Liver Transpl 14:1664-1670

Wanless I, Wong F, Blendis L et al (1995) Hepatic and portal vein thrombosis in cirrhosis: possible role in development of parenchymal extinction and portal hypertension. Hepatology 21:1238-1247

Warnaar NI, Molenaar IQ, Colquhoun SD et al (2008) Intraoperative pulmonary embolism and intracardiac thrombosis complicating liver transplantation: a systematic review. J Thromb Haemost 6:297-302

Warskulat U, Kreuels S, Muller HW et al (2001) Identification of osmosensitive and ammonia-regulated genes in rat astrocytes by Northern blotting and differential display reverse transcriptase-polymerase chain reaction. J Hepatol 35:358-366

Waters JH, Gottilieb A, Schoenwald P et al (2001) Normal saline versus lactated ringer's solution for intraoperative fluid management in patients undergoing abdominal aortic aneurysm repair: an outcome study. Anesth Analg 93:817-822

Wetzel RC, Setzer N, Stiff JL et al (1985) Hemodynamic responses in brain dead organ donor patients. Anesth Analg 64:125-128

Wiesner RH (1996) Is hepatic histology the true gold standard in diagnosing acute hepatic allograft rejection [editorial]? Liver Transpl Surg 2:165-167

Winnock S, Janvier G, Parmentier F et al (1993) Pontine myelinolysis following liver transplantation: a report of two cases. Transplant Int 6:26-28

Xia VW, Worapot A, Huang S et al (2015) Postoperative atrial fibrillation in liver transplantation. Am J Transplant 15:687-694

Yamaguchi H, Suzuki T, Shimura Y, et al (2015) Successful anesthetic management of a patient with critical bleeding during hepatectomy using recombinant activated factor VII and intraoperative blood salvage. Masui 64:666-670

Yokoyama I, Todo S, Miyata T et al (1989) Endotoxemia and human liver transplantation. Transplant Proc 21:3833-3841 